\title{
FEYNMAN-KAC FORMULA FOR ITERATED DERIVATIVES OF THE PARABOLIC ANDERSON MODEL
}

\author{
SEFIKA KUZGUN AND DAVID NUALART
}

\begin{abstract}
The purpose of this paper is to establish a Feynman-Kac formula for the moments of the iterated Malliavin derivatives of the solution to the parabolic Anderson model in terms of pinned Brownian motions. As an application, we obtain estimates for the moments of the iterated derivatives of the solution.
\end{abstract}

Mathematics Subject Classifications (2010): 60H07, 60H15.

Keywords: Feynman-Kac formula, stochastic heat equation, Malliavin calculus.

\section{INTRODUCTION}

Consider the following stochastic heat equation (SHE) also referred as the parabolic Anderson model (PAM):

$$
\frac{\partial u}{\partial t}=\frac{1}{2} \Delta u+u \dot{W}, \quad x \in \mathbb{R}^{d}, t \in(0, \infty)
$$

where $d \geq 1$, and $\Delta=\sum_{i=1}^{d} \frac{\partial^{2}}{\partial x_{i}^{2}}$ is the Laplacian operator. We assume that $\dot{W}$ is a centered Gaussian noise that is white in time and has an homogeneous covariance in the space variable. This is to say, informally,

$$
\mathrm{E}\left(\dot{W}\left(t_{1}, x_{1}\right) \dot{W}\left(t_{2}, x_{2}\right)\right)=\delta_{0}\left(t_{1}-t_{2}\right) \Lambda\left(x_{1}-x_{2}\right),
$$

where $\delta_{0}$ is the Dirac delta measure at zero, and $\Lambda$ is a non-negative definite tempered Borel measure on $\mathbb{R}^{d}$, satisfying Dalang's condition (2.2). The initial condition $u_{0}$ is assumed to be a signed Borel measure on $\mathbb{R}^{d}$ such that for all $c>0$,

$$
\int_{\mathbb{R}^{d}} e^{-c|x|^{2}}\left|u_{0}\right|(d x)<\infty .
$$

In particular, the initial data $u_{0}$ could be the Dirac measure $\delta_{0}$.

There is an extensive literature on the stochastic heat equation driven by an homogeneous noise. The existence and uniqueness of a mild solution to a nonlinear equation (with the noise multiplied by a Lipschitz function $\sigma(u)$ ) was first proved in [10] when $u_{0}$ is a bounded function, and was extended to the case where $u_{0}$ satisfies (1.2) in [1] for the case where the noise is a space-time white noise in $d=1$, and in [9] to the case where noise is white in time and satisfies Dalang's condition in space. Later the Hölder continuity in the space and time variables were established in [19, 2]. Using techniques of Malliavin calculus, several authors studied regularity properties of the density of the solution; see, for instance [7, 9]. When $\sigma(u)=u$, that means for equation (1.1), and $u_{0}$ is a bounded function, Feynman-Kac formulas for the solution and its moments were derived in [11.

Recently, assuming $u_{0}=1$, the Malliavin-Stein methodology has been applied to establish ergodicity in the space variable and to derive quantitative central limit theorems for spatial averages, see [3, 4, 15, 16]. A basic ingredient for these results is the fact that the $p$-norm of the Malliavin derivative of the solution at a given point, $\left\|D_{s, y} u(t, x)\right\|_{p}$ is bounded by a constant times the fundamental solution $p_{t-s}(x-y)$ to the heat equation. Motivated by these applications, the aim of this paper is to establish a Feynman-Kac formula for the moments of the iterated derivatives of the solution $u(t, x)$ to equation (1.1) and to derive moment estimates. 
Let us introduce some notation. For any integer $N \geq 1, D_{\boldsymbol{r}_{N}, \boldsymbol{z}_{N}}^{N} u(t, x)$ denotes the $N$-th iterated derivative of $u(t, x)$ in the sense of Malliavin calculus, that is

$$
D_{\boldsymbol{r}_{N}, \boldsymbol{z}_{N}}^{N} u(t, x)=D_{r_{1}, z_{1}} \cdots D_{r_{N}, z_{N}} u(t, x),
$$

for any $z_{1}, \ldots, z_{N} \in \mathbb{R}^{d}$ and $0<r_{1}<\cdots<r_{N}$ and with the notation $\boldsymbol{r}_{N}=\left(r_{1}, \ldots, r_{N}\right)$ and $z_{N}=\left(z_{1}, \ldots, z_{N}\right)$. We will denote by $p_{t}(x)$ the $d$-dimensional heat kernel:

$$
p_{t}(x)=(2 \pi t)^{-d / 2} \exp \left(-|x|^{2} /(2 t)\right), \quad x \in \mathbb{R}^{d}, t>0 .
$$

Our main result, stated below, provides an explicit formula for the moments of order $k$ of the iterated Malliavin derivatives of $u(t, x)$ in terms of the expectation of an exponential functional of a $k$-dimensional pinned Brownian motion.

Theorem 1.1. Let $u$ be the unique solution of (1.1) with initial condition $u_{0}$ which is a signed Borel measure satisfying (1.2). Let $N \geq 1$ be an integer and $z_{1}, \ldots, z_{N} \in \mathbb{R}^{d}, 0<r_{1}<\cdots<$ $r_{N}<t$. Then for integer $k \geq 2$, we have

$$
\begin{aligned}
\mathrm{E}\left[\left(D_{\boldsymbol{r}_{N}, \boldsymbol{z}_{N}}^{N} u(t, x)\right)^{k}\right]= & {\left[\prod_{m=1}^{N-1} p_{r_{m+1}-r_{m}}\left(z_{m+1}-z_{m}\right)\right]^{k} p_{t-r_{N}}^{k}\left(x-z_{N}\right) } \\
& \times \int_{\mathbb{R}^{k d}} \prod_{j=1}^{k} u_{0}\left(d \theta^{j}\right) \prod_{j=1}^{k} p_{r_{1}}\left(z_{1}-\theta^{j}\right) \\
& \times \mathrm{E}\left(\exp \left(\sum_{1 \leq j<l \leq k} \int_{0}^{t} \Lambda\left(\widehat{B}_{0, \boldsymbol{t}-\boldsymbol{r}_{N}, t}^{j, x, \boldsymbol{z}_{N}, \theta^{j}}(s)-\widehat{B}_{0, \boldsymbol{t}-\boldsymbol{r}_{N}, t}^{l, \boldsymbol{z}_{N}, \theta^{l}}(s)\right) d s\right)\right),
\end{aligned}
$$

where $\boldsymbol{t}-\boldsymbol{r}_{N}=\left(t-r_{1}, \ldots, t-r_{N}\right)$ and $\widehat{B}_{0, t-\boldsymbol{r}_{N}, t}^{j, x, z_{N}, \theta^{j}}, j=1, \ldots, k$ are independent d-dimensional pinned Brownian motions starting from $x$ with each component pinned at times $t-r_{m}$ to the points $z_{m}$ for $1 \leq m \leq N$, and pinned at $\theta^{j}$ at time $t$.

Moreover,

$$
\mathrm{E}\left(\exp \left(\sum_{1 \leq j<l \leq k} \int_{0}^{t} \Lambda\left(\widehat{B}_{0, t-\boldsymbol{r}_{N}, t}^{j, x, z_{N}, \theta^{j}}(s)-\widehat{B}_{0, \boldsymbol{t}-\boldsymbol{r}_{N}, t}^{l, x, z_{N}, \theta^{l}}(s)\right) d s\right)\right) \leq C_{t, k}
$$

where $C_{t, k}$ is a constant depending only on $t$ and $k$.

In the above theorem, taking into account that $\Lambda$ might be a measure, the composition $\Lambda\left(\widehat{B}_{0, \boldsymbol{t}-\boldsymbol{r}_{N}, t}^{j, x, \boldsymbol{z}_{N}, \theta^{j}}(s)-\widehat{B}_{0, \boldsymbol{t}-\boldsymbol{r}_{N}, t}^{l, x, \boldsymbol{z}_{N}, \theta^{l}}(s)\right)$ needs to be properly defined as a limit in $L^{2}(\Omega)$, using an approximation argument, see Proposition 3.1, part (ii). When $\Lambda(d x)=\Lambda(x) d x$, then this is just an ordinary composition of the density $\Lambda$ with the random variable $\widehat{B}_{0, \boldsymbol{t}-\boldsymbol{r}_{N}, t}^{j, x, \boldsymbol{z}_{N}, \theta^{j}}(s)-\widehat{B}_{0, \boldsymbol{t}-\boldsymbol{r}_{N}, t}^{l, x, \boldsymbol{z}_{N}, \theta^{l}}(s)$.

As a consequence of Theorem 1.1, we deduce the following result.

Corollary 1.2. Under the assumptions and notation of Theorem 1.1, we have

$$
\left\|D_{\boldsymbol{r}_{N}, \boldsymbol{z}_{N}}^{N} u(t, x)\right\|_{k} \leq C_{t, k}^{1 / k}\left(p_{r_{1}} *\left|u_{0}\right|\right)\left(z_{1}\right)\left(\prod_{m=1}^{N-1} p_{r_{m+1}-r_{m}}\left(z_{m+1}-z_{m}\right)\right) p_{t-r_{N}}\left(x-z_{N}\right) .
$$

Here is a survey of previous results related to Corollary [1.2, obtained by alternative methods. The estimate (1.4) for $N=1$ was first proved in [15, Lemma 5.1] for the nonlinear stochastic heat equation with $u_{0}=1$, driven by a space-time white noise, and later extended to the case of a Riesz-type covariance in [16, Lemma 2.1] and to the case of a general spatial covariance satisfying (2.2) in [3, Theorem 4.6]. In the paper [6], the authors have also considered the case $N=2$, but only for the parabolic Anderson model (1.4), the case of a general coefficient $\sigma$ being an open problem. Finally, in the case of equation (1.1) with $u_{0}=\delta_{0}$, the estimate (1.4) for $N=1$ has been obtained in [5, Lemma 2.1].

The paper is organized as follows. After some preliminaries in Section 2, Section 3 is devoted to establishing a Feynman-Kac formula for the solution to equation (1.1) driven by a regularization 
$W^{\epsilon}$ of the noise. In this section we also derive a Feynman-Kac formula for the moments of the solution to equation (1.1). Finally, Section 4 contains the proof of Theorem 1.1.

\section{Preliminaries}

2.1. The Set-Up. Firstly, we introduce some notation. The space of Schwartz functions, that is, the space of infinitely differentiable rapidly decreasing functions on $\mathbb{R}_{+} \times \mathbb{R}^{d}$, respectively on $\mathbb{R}^{d}$, will be denoted by $\mathscr{S}\left(\mathbb{R}_{+} \times \mathbb{R}^{d}\right)$, respectively by $\mathscr{S}\left(\mathbb{R}^{d}\right)$. Then $\mathscr{S}^{\prime}\left(\mathbb{R}^{d}\right)$ will denote the dual of $\mathscr{S}\left(\mathbb{R}^{d}\right)$ and its elements are the so-called tempered distributions. The Fourier transform of a tempered Borel measure $\nu$ on $\mathbb{R}^{d}$ is defined by

$$
\mathscr{F}(\nu)(\xi)=\int_{\mathbb{R}^{d}} e^{-i \xi \cdot x} \nu(d x),
$$

which is a slowly increasing $C^{\infty}$ function.

Let $(\Omega, \mathcal{F}, P)$ be a complete probability space, and $W$ a Gaussian noise encoded by a centered Gaussian family $\left\{W(\varphi) ; \varphi \in \mathscr{S}\left(\mathbb{R}_{+} \times \mathbb{R}^{d}\right)\right\}$ with the covariance structure

$$
E(W(\varphi) W(\psi))=\left\langle\varphi, \psi *\left(\delta_{0} \times \Lambda\right)\right\rangle_{L^{2}\left(\mathbb{R}_{+} \times \mathbb{R}^{d}\right)},
$$

where $\Lambda$ is a nonnegative definite tempered Borel measure on $\mathbb{R}^{d}$. We assume that the Fourier transform of $\Lambda$, denoted by $\mathscr{F} \Lambda=\mu$, is a measure satisfying Dalang's condition:

$$
\int_{\mathbb{R}^{d}} \frac{\mu(d \xi)}{1+|\xi|^{2}}<\infty
$$

Let $\mathcal{H}$ be the completion of $\mathscr{S}\left(\mathbb{R}_{+} \times \mathbb{R}^{d}\right)$ endowed with the inner product

$$
\begin{aligned}
\langle\varphi, \psi\rangle_{\mathcal{H}} & =\left\langle\varphi, \psi *\left(\delta_{0} \times \Lambda\right)\right\rangle_{L^{2}\left(\mathbb{R}_{+} \times \mathbb{R}^{d}\right)} \\
& =\int_{0}^{\infty} \int_{\mathbb{R}^{2 d}} \varphi(s, y) \psi\left(s, y-y^{\prime}\right) \Lambda\left(d y^{\prime}\right) d y d s \\
& =\int_{0}^{\infty} \int_{\mathbb{R}^{d}} \mathscr{F} \varphi(s, \cdot)(\xi) \overline{\mathscr{F} \psi(s, \cdot)(\xi)} \mu(d \xi) d s .
\end{aligned}
$$

The mapping $\varphi \mapsto W(\varphi)$ defined on $\mathscr{S}\left(\mathbb{R}_{+} \times \mathbb{R}^{d}\right)$ can be extended to a linear isometry between $\mathcal{H}$ and the Gaussian space spanned by $W$. This isometry will be denoted by

$$
W(\varphi)=\int_{0}^{\infty} \int_{\mathbb{R}^{d}} \varphi(s, y) W(d s, d y)
$$

for $\varphi \in \mathcal{H}$. With this notation in mind, we have $\mathrm{E}(W(\varphi) W(\psi))=\langle\varphi, \psi\rangle_{\mathcal{H}}$. We will use the notation $W(t, x)$ as shorthand for $W\left(\mathbb{1}_{[0, t] \times[0, x]}\right)$, so that we can write

$$
\begin{aligned}
\mathrm{E}\left(W(t, x) W\left(t^{\prime}, x^{\prime}\right)\right) & =\left(t \wedge t^{\prime}\right) \int_{\mathbb{R}^{2 d}} \mathbb{1}_{[0, x]}(y) \mathbb{1}_{\left[0, x^{\prime}\right]}\left(y-y^{\prime}\right) \Lambda\left(d y^{\prime}\right) d y \\
& =\left(t \wedge t^{\prime}\right) \int_{\mathbb{R}^{d}} \mathscr{F} \mathbb{1}_{[0, x]}(\cdot)(\xi) \overline{\mathscr{F} \mathbb{1}_{\left[0, x^{\prime}\right]}(\cdot)(\xi)} \mu(d \xi) .
\end{aligned}
$$

Let $\mathcal{H}_{0}$ be the completion of $\mathscr{S}\left(\mathbb{R}^{d}\right)$ with the inner product

$$
\left\langle\phi_{1}, \phi_{2}\right\rangle_{\mathcal{H}_{0}}=\int_{\mathbb{R}^{2 d}} \phi_{1}(y) \phi_{2}\left(y-y^{\prime}\right) \Lambda\left(d y^{\prime}\right) d y .
$$

In this way, we have $\mathcal{H}=L^{2}\left(\mathbb{R}_{+} ; \mathcal{H}_{0}\right)$.

For each $t \geq 0$, let $\mathcal{F}_{t}$ be the $\sigma$-field generated by the random variables $W(\varphi)$ where $\varphi$ has support in $[0, t] \times \mathbb{R}^{d}$. We say that a random field $u=\left\{u(t, x) ;(t, x) \in[0, \infty) \times \mathbb{R}^{d}\right\}$ is adapted if for each $(t, x) \in[0, \infty) \times \mathbb{R}^{d}$ the random variable $u(t, x)$ is $\mathcal{F}_{t}$ measurable. 
Definition 2.1. A random field $u=\left\{u(t, x) ;(t, x) \in[0, \infty) \times \mathbb{R}^{d}\right\}$ is called a mild solution to (1.1) with initial condition $u_{0}$, if $u$ is adapted, jointly measurable with respect to $\mathcal{B}\left([0, \infty) \times \mathbb{R}^{d}\right) \times$ $\mathcal{F}, \mathrm{E}\left(u(t, x)^{2}\right)<\infty$ for all $(t, x) \in(0, \infty) \times \mathbb{R}^{d}$ and $u$ satisfies the integral equation

$$
u(t, x)=\left(p_{t} * u_{0}\right)(x)+\int_{0}^{t} \int_{\mathbb{R}^{d}} p_{t-s}(x-y) u(s, y) W(d s, d y), \quad \text { a.s. }
$$

for all $(t, x) \in 0, \infty) \times \mathbb{R}^{d}$, where the above stochastic integral is to be understood in the sense defined by Walsh in [20] and extended by Dalang in [10].

2.2. Malliavin Calculus. In this subsection we will recall some basic elements of the Malliavin calculus associated with $W$. For more details on the Malliavin calculus, we refer to [18, 17]. For a smooth and cylindrical random variable $F=f\left(W\left(\varphi_{1}\right), \ldots, W\left(\varphi_{n}\right)\right)$, with $\varphi_{i} \in \mathcal{H}, 1 \leq i \leq n$ and $f \in C_{b}^{\infty}\left(\mathbb{R}^{n}\right)$ ( $f$ and all its partial derivatives are bounded), we define its Malliavin derivative as an $\mathcal{H}$-valued random variable defined as

$$
D F=\sum_{i=1}^{n} \frac{\partial f}{\partial x_{i}}\left(W\left(\varphi_{1}\right), \ldots, W\left(\varphi_{n}\right)\right) \varphi_{i} .
$$

By iteration, we can also define the $k$-th derivative $D^{k} F$, which is an element in the space $L^{2}\left(\Omega ; \mathcal{H}^{\otimes k}\right)$. For any real $p \geq 1$ and any integer $k \geq 1$, the Sobolev space $\mathbb{D}^{k, p}$ is defined as the closure of the space of smooth and cylindrical random variables with respect to the norm $\|\cdot\|_{k, p}$ defined by

$$
\|F\|_{k, p}^{p}=\mathbb{E}\left(|F|^{p}\right)+\sum_{i=1}^{k} \mathbb{E}\left(\left\|D^{i} F\right\|_{\mathcal{H}^{\otimes i}}^{p}\right) .
$$

We set $\mathbb{D}^{\infty}:=\cap_{k, p \in \mathbb{N}} \mathbb{D}^{k, p}$.

The following result ensures on the regularity of the solution to equation (1.1) in the sense of Malliavin calculus.

Proposition 2.1. For any $(t, x) \in(0, \infty) \times \mathbb{R}^{d}, u(t, x) \in \mathbb{D}^{\infty}$.

Proof. From Part (2) of [9, Proposion 3.2] it follows that $u(t, x) \in \mathbb{D}^{1, p}$ for all $(t, x) \in(0, \infty) \times \mathbb{R}^{d}$ and for all $p \geq 1$. Because we are dealing with the parabolic Anderson model, the proof of Part (3) of [9, Proposion 3.2] implies that $u(t, x) \in \mathbb{D}^{\infty}$ for all $(t, x) \in(0, \infty) \times \mathbb{R}^{d}$.

2.3. Brownian bridges. Along the paper, $\left\{\widehat{B}_{[a, b]}^{x, y}(s) ; s \in[a, b]\right\}$ will denote a $d$-dimensional Brownian bridge in the time interval $[a, b]$ that goes from the starting point $x$ at time $a$ to the end point $y$ at time $b$. We also set $\widehat{B}_{[a, b]}:=\widehat{B}_{[a, b]}^{0,0}$. We recall that the Brownian bridge $\widehat{B}_{[a, b]}^{x, y}$ can be expressed as

$$
\widehat{B}_{[a, b]}^{x, y}(s)=\widehat{B}_{[a, b]}(s)+\frac{s-a}{b-a} y+\frac{b-s}{b-a} x, \quad x, y \in \mathbb{R}^{d} .
$$

\section{Regularization of the Noise}

We will introduce the following regularization of the noise $W$ in the space variable. For each $\epsilon>0$ and any $\varphi \in \mathscr{S}\left(\mathbb{R}_{+} \times \mathbb{R}^{d}\right)$, we define

$$
W^{\epsilon}(\varphi)=W\left(\varphi * p_{\epsilon}\right),
$$

where $*$ denotes the convolution in the space variable and $p_{\epsilon}(x)$ is the $d$-dimensional heat kernel defined in (1.3). Then, the Gaussian family $W^{\epsilon}=\left\{W^{\epsilon}(\varphi) ; \varphi \in \mathscr{S}\left(\mathbb{R}_{+} \times \mathbb{R}^{d}\right)\right\}$ has the covariance structure

$$
\begin{aligned}
\mathrm{E}\left(W^{\epsilon}(\varphi) W^{\epsilon}(\psi)\right) & =\int_{0}^{\infty} \int_{\mathbb{R}^{2 d}}\left(\varphi(s, \cdot) * p_{\epsilon}(\cdot)\right)(y)\left(\psi(s, \cdot) * p_{\epsilon}(\cdot)\right)\left(y-y^{\prime}\right) \Lambda\left(d y^{\prime}\right) d y d s \\
& =\int_{0}^{\infty} \int_{\mathbb{R}^{d}} \mathscr{F}(\varphi)(s, \xi) \overline{\mathscr{F}(\psi)(s, \xi)} e^{-\epsilon|\xi|^{2}} \mu(d \xi) d s,
\end{aligned}
$$


that is, the noise $W^{\epsilon}$ is white in time and it has a spatial covariance given by

$$
\Lambda_{\epsilon}(x)=\frac{1}{(2 \pi)^{d}} \int_{\mathbb{R}^{d}} e^{i x \cdot \xi-\epsilon|\xi|^{2}} \mu(d \xi)
$$

whose Fourier transform is $\mu_{\epsilon}(d \xi)=e^{-\epsilon|\xi|^{2}} \mu(d \xi)$. Notice that $\mu_{\epsilon}$ is a finite measure and $\Lambda_{\epsilon}$ is a bounded smooth function. In this way, we can write

$$
\begin{aligned}
\mathrm{E}\left(W^{\epsilon}(\varphi) W^{\epsilon}(\psi)\right) & =\int_{0}^{\infty} \int_{\mathbb{R}^{2 d}} \varphi(s, y) \psi\left(s, y^{\prime}\right) \Lambda_{\epsilon}\left(y-y^{\prime}\right) d y d y^{\prime} d s \\
& =\int_{0}^{\infty} \int_{\mathbb{R}^{d}} \mathscr{F}(\varphi)(s, \xi) \overline{\mathscr{F}(\psi)(s, \xi)} \mu_{\epsilon}(d \xi) d s .
\end{aligned}
$$

As before, we denote by $\mathcal{H}^{\epsilon}$ the completion of $\mathscr{S}\left(\mathbb{R}_{+} \times \mathbb{R}^{d}\right)$ under the inner product

$$
\langle\varphi, \psi\rangle_{\mathcal{H}^{\epsilon}}=\mathrm{E}\left(W^{\epsilon}(\varphi) W^{\epsilon}(\psi)\right) .
$$

For a fixed $t \in \mathbb{R}_{\geq 0}$, let $f^{t}:[0, t] \rightarrow \mathbb{R}^{d}$ be a continuous function. Then, the map $(s, y) \mapsto$ $\mathbb{1}_{[0, t]}(s) p_{\epsilon}\left(f^{t}(s)-y\right)$ belongs to the space $\mathcal{H}$ since

$$
\begin{aligned}
\left\|\mathbb{1}_{[0, t]}(\bullet) p_{\epsilon}\left(f^{t}(\bullet)-\star\right)\right\|_{\mathcal{H}}^{2} & =\int_{0}^{t} \int_{\mathbb{R}^{2 d}} p_{\epsilon}\left(f^{t}(s)-y\right) p_{\epsilon}\left(f^{t}(s)-y^{\prime}+y\right) \Lambda\left(d y^{\prime}\right) d y d s \\
& =\int_{0}^{t} \int_{\mathbb{R}^{d}} e^{-\epsilon|\xi|^{2}} \mu(d \xi) d s=t \int_{\mathbb{R}^{d}} e^{-\epsilon|\xi|^{2}} \mu(d \xi) \\
& =t(2 \pi)^{d} \Lambda_{\epsilon}(0)<\infty
\end{aligned}
$$

and we can define the stochastic integral

$$
W\left(\mathbb{1}_{[0, t]}(\bullet) p_{\epsilon}\left(f^{t}(\bullet)-\star\right)\right)=\int_{0}^{t} \int_{\mathbb{R}^{d}} p_{\epsilon}\left(f^{t}(s)-y\right) W(d s, d y) .
$$

Throughout, we will use the following notation:

$$
\int_{0}^{t} W^{\epsilon}\left(d s, f^{t}(s)\right):=\int_{0}^{t} \int_{\mathbb{R}^{d}} p_{\epsilon}\left(f^{t}(s)-y\right) W(d s, d y) .
$$

From (3.2) it follows that $\int_{0}^{t} W^{\epsilon}\left(d s, f^{t}(s)\right)$ is a centered Gaussian random variable with variance $t(2 \pi)^{d} \Lambda_{\epsilon}(0)$.

The following result will play an important role in the proof of our main result.

Proposition 3.1. Fix an integer $k \geq 2$. Let $B_{[a, b]}^{j}, j=1, \ldots, k$ be independent d-dimensional Brownian bridges in $[a, b]$ from 0 to 0 , where $[a, b] \subset[0, t]$. Consider a measurable function $\alpha=\left(\alpha^{j, l}\right)_{1 \leq j<l \leq k}:[a, b] \rightarrow \mathbb{R}^{k(k-1) / 2}$. For each $1 \leq j<l \leq k$ we set

$$
G_{\epsilon}^{j, l}:=\int_{a}^{b} \Lambda_{\epsilon}\left(B_{[a, b]}^{j}(s)-B_{[a, b]}^{l}(s)+\alpha^{j, l}(s)\right) d s .
$$

Then the following results hold true:

(i) For each $\kappa \in \mathbb{R}$,

$$
\sup _{\epsilon \in(0,1]} \sup _{\alpha} \mathrm{E}\left(\exp \left(\kappa \sum_{1 \leq j<l \leq k} G_{\epsilon}^{j, l}\right)\right)=K_{t, \kappa}<\infty,
$$

where the constant $K_{t, \kappa}$ only depends on $t$ and $\kappa$.

(ii) For each $1 \leq j<l \leq k$, the random variables $G_{\epsilon}^{j, l}$ converge in $L^{p}(\Omega)$ for all $p \geq 2$, as $\epsilon \downarrow 0$, to a limit denoted by $G^{j, l}:=\int_{a}^{b} \Lambda\left(B_{[a, b]}^{j}(s)-B_{[a, b]}^{l}(s)+\alpha^{j, l}(s)\right) d s$. 
(iii) We have, for all $\kappa \in \mathbb{R}$,

$$
\lim _{\epsilon \downarrow 0} \mathrm{E}\left(\exp \left(\kappa \sum_{1 \leq j<l \leq k} G_{\epsilon}^{j, l}\right)\right)=\mathrm{E}\left(\exp \left(\kappa \sum_{1 \leq j<l \leq k} G^{j, l}\right)\right),
$$

where the convergence is uniform in $\alpha$ and in $a, b$.

Proof. Property (3.3) has been proved in [13, Lemma 4.1]. The convergence in point (iii), as $\epsilon$ tends to zero, follows from [13, Proposition 4.2]. Actually, in Proposition 4.2 of [13, the result is proved for $\alpha^{j, l}(s)=x^{j}-x^{l}$, where $x^{j} \in \mathbb{R}^{d}, j=1, \ldots, k$, but the case of a general function $\alpha$ can be done in the same way. Property (ii) is proved in Proposition 4.3 of 13 for Brownian motions and with $\alpha^{j, l}(s)=x^{j}-x^{l}$ and the arguments of the proof are also valid for Brownian bridges and for a general function $\alpha$.

Now, we consider the heat equation driven by $W^{\epsilon}$,

$$
\frac{\partial u}{\partial t}=\frac{1}{2} \Delta u+u \dot{W}^{\epsilon}, \quad x \in \mathbb{R}^{d}, t \in \mathbb{R}_{+},
$$

with initial condition $u(0, x)=u_{0}$ where $u_{0}$ is a signed Borel measure satisfying (1.2). An adapted and jointly measurable random field $u^{\epsilon}=\left\{u^{\epsilon}(t, x) ;(t, x) \in(0, \infty) \times \mathbb{R}^{d}\right\}$ such that $\mathrm{E}\left(u^{\epsilon}(t, x)\right)^{2}<\infty$ for all $(t, x) \in(0, \infty) \times \mathbb{R}^{d}$ is a mild solution to equation (3.4), if for any $(t, x) \in(0, \infty) \times \mathbb{R}^{d}$, the process $\left\{p_{t-s}(x-y) u^{\epsilon}(s, y) \mathbb{1}_{[0, t]}(s) ;(s, y) \in(0, \infty) \times \mathbb{R}^{d}\right\}$ is integrable with respect to $W^{\epsilon}$, and the following holds:

$$
u^{\epsilon}(t, x)=\left(p_{t} * u_{0}\right)(x)+\int_{0}^{t} \int_{\mathbb{R}^{d}} p_{t-s}(x-y) u^{\epsilon}(s, y) W^{\epsilon}(d s, d y) .
$$

It follows from the general theory that this mild solution exists and it is unique. Furthermore, because the spectral measure is finite, there is a Feynman-Kac representation of the solution, given in the following lemma. For the sake of completeness we include a proof of the lemma, based on a duality argument.

Lemma 3.1. For each $\epsilon>0$, the following random field $u^{\epsilon}(t, x)$ is the solution to the heat equation given in (3.4):

$$
u^{\epsilon}(t, x)=\mathrm{E}^{B}\left(u_{0}\left(B_{t}^{x}\right) \exp \left(\int_{0}^{t} W^{\epsilon}\left(d s, B_{t-s}^{x}\right)-\frac{1}{2} t(2 \pi)^{d} \Lambda_{\epsilon}(0)\right)\right),
$$

where $B^{x}$ is a d-dimensional standard Brownian motion independent of $W$ that starts at $x$ and $\mathrm{E}^{B}$ denotes the mathematical expectation with respect to $B^{x}$.

Remark 1. Notice that, because $u_{0}$ is a signed measure, the composition $u_{0}\left(B_{t}^{x}\right)$ is not well defined. The right-hand side of equation (3.6), can be interpreted in two ways:

(i) We can write

$$
\left.u^{\epsilon}(t, x)=\int_{\mathbb{R}^{d}} u_{0}(d \theta) p_{t}(x-\theta)\right) \mathrm{E}^{\widehat{B}}\left(\exp \left(\int_{0}^{t} \int_{\mathbb{R}^{d}} p_{\epsilon}\left(\widehat{B}_{0, t}^{\theta, x}(s)-y\right) W(d s, d y)-\frac{1}{2} t(2 \pi)^{d} \Lambda_{\epsilon}(0)\right)\right),
$$

where $\left\{\widehat{B}_{0, t}^{\theta, x}(s), s \in[0, t]\right\}$ denotes a $d$-dimensional Brownian bridge in the interval $[0, t]$ from $\theta$ to $x$. The above integral is well defined almost surely because on one hand $\left.\int_{\mathbb{R}^{d}}\left|u_{0}\right|(d \theta) p_{t}(x-\theta)\right)<$ $\infty$ and moreover, from the computations in (4.3), we have

$$
\mathrm{E}^{W} \mathrm{E}^{\widehat{B}}\left(\exp \left(\int_{0}^{t} \int_{\mathbb{R}^{d}} p_{\epsilon}\left(\widehat{B}_{0, t}^{\theta, x}(s)-y\right) W(d s, d y)\right)\right)=e^{\frac{1}{2} t(2 \pi)^{d} \Lambda_{\epsilon}(0)} .
$$

(ii) From the results in [7] the random variable $u_{0}\left(B_{t}^{x}\right)$ belongs to Meyer-Watanabe space $\mathbb{D}^{-\alpha, p}$ for any $p>1$ and $\alpha>1-\frac{1}{p}$. Furthermore, it can be proved that, conditionally to $W$, the 
exponential term $\mathcal{E}:=\exp \left(\int_{0}^{t} \int_{\mathbb{R}^{d}} p_{\epsilon}\left(B_{t-s}^{x}-y\right) W(d s, d y)\right)$ is in the space $\mathbb{D}^{1,2}$ as a functional of the Brownian motion $B$, with a derivative given by

$$
D_{r} \mathcal{E}=\mathcal{E} \sum_{i=1}^{d} \int_{0}^{t-r} \int_{\mathbb{R}^{d}} \frac{\partial p_{\epsilon}}{\partial x_{i}}\left(B_{t-s}^{x}-y\right) W(d s, d y) .
$$

Then, the right-hand side of equation (3.6) can be expressed as the following pairing

$$
e^{-\frac{1}{2} \Lambda_{\epsilon}(0)}\left\langle u_{0}\left(B_{t}^{x}\right), \mathcal{E}\right\rangle_{\mathbb{D}^{-1,2}, \mathbb{D}^{1,2}} .
$$

Proof of Lemma 3.1. Let $G \in L^{2}(\Omega, \mathcal{F}, P)$ be such that $G=e^{W(h)-\frac{1}{2}\|h\|_{\mathcal{H}}^{2}}$ for some $h \in \mathcal{H}$. From (3.6), we obtain

$$
\begin{aligned}
\mathrm{E}\left(G u^{\epsilon}(t, x)\right) & =\mathrm{E}^{W}\left(G E^{B}\left(u_{0}\left(B_{t}^{x}\right) \exp \left(\int_{0}^{t} W^{\epsilon}\left(d s, B_{t-s}^{x}\right)-\frac{1}{2} t(2 \pi)^{d} \Lambda_{\epsilon}(0)\right)\right)\right) \\
& =\mathrm{E}^{B}\left(u_{0}\left(B_{t}^{x}\right) \mathrm{E}^{W}\left(\exp \left(W\left(h+p_{\epsilon}\left(B_{t-\bullet}^{x}-\star\right)\right)-\frac{1}{2}\|h\|_{\mathcal{H}}^{2}-\frac{1}{2} t(2 \pi)^{d} \Lambda_{\epsilon}(0)\right)\right)\right) \\
& =\mathrm{E}^{B}\left(u_{0}\left(B_{t}^{x}\right) \exp \left(\frac{1}{2}\left\|h+p_{\epsilon}\left(B_{t-\bullet}^{x}-\star\right)\right\|_{\mathcal{H}}^{2}-\frac{1}{2}\|h\|_{\mathcal{H}}^{2}-\frac{1}{2} t(2 \pi)^{d} \Lambda_{\epsilon}(0)\right)\right) \\
& =\mathrm{E}^{B}\left(u_{0}\left(B_{t}^{x}\right) \exp \left(\left\langle p_{\epsilon}\left(B_{t-\bullet}^{x}-\star\right), h\right\rangle_{\mathcal{H}}^{2}\right)\right) \\
& =\mathrm{E}^{B}\left(u_{0}\left(B_{t}^{x}\right) \exp \left(\int_{0}^{t}\left\langle p_{\epsilon}\left(B_{t-s}^{x}-\star\right), h(s, \star)\right\rangle_{\mathcal{H}_{0}} d s\right)\right) .
\end{aligned}
$$

Letting $S_{t, x}(h)=\mathrm{E}^{W}\left(G u^{\epsilon}(t, x)\right)$, by the classical Feynmann-Kac's formula, the above calculation shows that $S_{t, x}(h)$ satisfies the classical heat equation with potential $\left\langle p_{\epsilon}(x-\star), h(s, \star)\right\rangle_{\mathcal{H}_{0}}$, and initial condition $u_{0}$, i.e.

$$
\frac{\partial S_{t, x}(h)}{\partial t}=\frac{1}{2} \Delta S_{t, x}(h)+S_{t, x}(h)\left\langle p_{\epsilon}(x-\star), h(t, \star)\right\rangle_{\mathcal{H}_{0}}
$$

As a consequence, we have

$$
\begin{aligned}
S_{t, x}(h) & =\left(p_{t} * u_{0}\right)(x)+\int_{0}^{t} \int_{\mathbb{R}^{d}} p_{t-s}(x-y) S_{s, y}(h)\left\langle p_{\epsilon}(y-\star), h(s, \star)\right\rangle_{\mathcal{H}_{0}} d s d y \\
& =\left(p_{t} * u_{0}\right)(x)+\int_{0}^{t} \int_{\mathbb{R}^{d}} p_{t-s}(x-y) \mathrm{E}\left(u_{s, y}^{\epsilon}\left\langle p_{\epsilon}(y-\star), D_{s, \star} G\right\rangle_{\mathcal{H}_{0}}\right) d s d y,
\end{aligned}
$$

where we used $D G=h G$. In conclusion, we have proved that

$$
\mathrm{E}\left(G u^{\epsilon}(t, x)\right)=\left(p_{t} * u_{0}\right)(x)+\mathrm{E}\left(\left\langle\mathbb{1}_{[0, t]}(\bullet) \int_{\mathbb{R}^{d}} p_{t-\bullet}(x-y) p_{\epsilon}(y-\star) u^{\epsilon}(\bullet, y) d y, D G\right\rangle_{\mathcal{H}}\right) .
$$

By the fact that the Dalang-Walsh stochastic integral is the adjoint of the Malliavin derivative, we deduce that

$$
u^{\epsilon}(t, x)=\left(p_{t} * u_{0}\right)(x)+\int_{0}^{t} \int_{\mathbb{R}^{d}}\left(\int_{\mathbb{R}^{d}} p_{t-s}(x-y) p_{\epsilon}(y-z) u^{\epsilon}(s, y) d y\right) W(d s, d z),
$$

which implies equation (3.5).

In the next theorem we show that $u^{\epsilon}(t, x)$ converges to the solution $u(t, x)$ of the stochastic heat equation (1.1) in $L^{p}(\Omega)$ for all $p \geq 1$, and, as a consequence, we derive a Feynman-Kac formula for the moments of the solution. This type of Feynman-Kac formula has been established in the literature under different conditions (see, for instance, [11, Theorem 3.6] for the case where $\Lambda$ is a function and there is also a correlation in time, or [12] when the noise is white in space and a fractional Brownian motion with Hurst parameter $H>1 / 2$ in time) assuming that $u_{0}$ is a bounded function. We will give here a detailed proof based on the approximation scheme $u^{\epsilon}(t, x)$, because the necessary computations will be also used in the proof of Theorem 1.1 . 
Proposition 3.2. Let $u^{\epsilon}$ be the solution to equation (3.5) with an initial condition $u_{0}$ satisfying (1.2). Then, for any $k \geq 1$, we have

$$
\sup _{\epsilon>0} \mathrm{E}\left(\left|u^{\epsilon}(t, x)\right|^{k}\right)<\infty
$$

and the following convergence holds in $L^{p}(\Omega)$ for any $p \geq 1$ :

$$
\lim _{\epsilon \rightarrow 0} u^{\epsilon}(t, x)=u(t, x)
$$

where $u$ is the solution to the stochastic heat equation (1.1) with initial condition $u_{0}$. Furthermore, for any integer $k \geq 2$, the following Feynmann-Kac formula holds:

$$
\mathrm{E}\left[u^{k}(t, x)\right]=\mathrm{E}\left(\prod_{j=1}^{k} u_{0}\left(B_{t}^{j, x}\right) \exp \left(\sum_{1 \leq j<l \leq k} \int_{0}^{t} \Lambda\left(B_{s}^{j}-B_{s}^{l}\right) d s\right)\right)
$$

where $B=\left\{B^{j}\right\}_{j=1, \ldots, k}$ is an independent family of d-dimensional standard Brownian motions and the integrals $\int_{0}^{t} \Lambda\left(B_{s}^{j}-B_{s}^{l}\right) d s$ are defined according to Proposition 3.1 (ii).

Proof. Set $\Psi_{t, x}^{k}=: \prod_{j=1}^{k} u_{0}\left(B_{t}^{j, x}\right)$. Using Lemma 3.1, we have

$$
\mathrm{E}\left[\left(u^{\epsilon}(t, x)\right)^{k}\right]=\mathrm{E}^{W} \mathrm{E}^{B}\left(\Psi_{t, x}^{k} \exp \left(\sum_{j=1}^{k} \int_{0}^{t} W^{\epsilon}\left(d s, B_{t-s}^{j, x}\right)-\frac{1}{2} t(2 \pi)^{d} \Lambda_{\epsilon}(0)\right)\right),
$$

where $B=\left\{B^{j}\right\}_{j=1, \ldots, k}$ is a family of $d$-dimensional independent standard Brownian motions independent of $W$ and $B^{j, x}=B^{j}+x$. Here again the expectation in (3.10) has to be understood as in Remark 1. Changing the order of the expectations, yields

$$
\begin{aligned}
& \mathrm{E}\left[\left(u^{\epsilon}(t, x)\right)^{k}\right] \\
& =\mathrm{E}^{B}\left(\Psi_{t, x}^{k} \mathrm{E}^{W}\left(\exp \left(\sum_{j=1}^{k} \int_{0}^{t} \int_{\mathbb{R}^{d}} p_{\epsilon}\left(B_{t-s}^{j, x}-y\right) W(d s, d y)-\frac{1}{2} t(2 \pi)^{d} \Lambda_{\epsilon}(0)\right)\right)\right. \\
& =\mathrm{E}\left(\Psi_{t, x}^{k} \exp \left(\frac{1}{2} \sum_{j, l=1, j \neq l}^{k} \int_{0}^{t} \int_{\mathbb{R}^{2 d}} p_{\epsilon}\left(B_{t-s}^{j, x}-y\right) p_{\epsilon}\left(B_{t-s}^{l, x}-y+y^{\prime}\right) \Lambda\left(d y^{\prime}\right) d y d s\right)\right) \\
& =\mathrm{E}\left(\Psi_{t, x}^{k} \exp \left(\sum_{1 \leq j<l \leq k} \int_{0}^{t} \Lambda_{2 \epsilon}\left(B_{s}^{j, x}-B_{s}^{l, x}\right) d s\right)\right) .
\end{aligned}
$$

Integrating with respect to the law of the random vector $\left(B_{t}^{1}+x, \ldots, B_{t}^{k}+x\right)$ whose density is $\theta \mapsto \prod_{j=1}^{k} p_{t}\left(x-\theta_{j}\right)$, the above expectation can be written as follows

$$
\begin{aligned}
\mathrm{E}\left[\left(u^{\epsilon}(t, x)\right)^{k}\right]= & \int_{\mathbb{R}^{k d}} \prod_{j=1}^{k} u_{0}\left(d \theta_{j}\right) p_{t}\left(x-\theta_{j}\right) \\
& \times \mathrm{E}\left(\exp \left(\sum_{1 \leq j<l \leq k} \int_{0}^{t} \Lambda_{2 \epsilon}\left(\widehat{B}_{0, t}^{j, x, \theta_{j}}(s)-\widehat{B}_{0, t}^{l, x, \theta_{l}}(s)\right) d s\right)\right),
\end{aligned}
$$


where $\left\{\widehat{B}_{0, t}^{j, \theta_{j}, x}, j=1, \ldots k\right\}$ denote a family of $d$-dimensional Brownian bridges in the interval $[0, t]$ from $x$ to $\theta_{j}$. Now, using the expression (2.7) for Brownian bridges, we can write

$$
\begin{aligned}
\mathrm{E}\left[\left(u^{\epsilon}(t, x)\right)^{k}\right]= & \int_{\mathbb{R}^{k d}} \prod_{j=1}^{k} u_{0}\left(d \theta_{j}\right) p_{t}\left(x-\theta_{j}\right) \\
& \times \mathrm{E}\left(\exp \left(\sum_{1 \leq j<l \leq k} \int_{0}^{t} \Lambda_{2 \epsilon}\left(\widehat{B}_{0, t}^{j}(s)-\widehat{B}_{0, t}^{l}(s)+\frac{s\left(\theta_{j}-\theta_{l}\right)}{t}\right) d s\right)\right) .
\end{aligned}
$$

Now we can proceed with the proof of the proposition. First, we only need to show (3.7) when $k$ is even. In this case, (3.7) follows from formula (3.12), condition (1.2) and (3.3). Indeed, we have

$$
\mathrm{E}\left[\left(u^{\epsilon}(t, x)\right)^{k}\right] \leq c_{t}\left(\int_{\mathbb{R}^{d}}\left|u_{0}\right|(d \theta) p_{t}(x-\theta)\right)^{k}<\infty,
$$

where $c_{t}$ is a finite constant only depending on $t$.

We claim that $u^{\epsilon}(t, x)$ converges in $L^{p}(\Omega)$ as $\epsilon \rightarrow 0$, for all $p \geq 2$. Indeed,

$$
\begin{aligned}
\mathrm{E}\left(u^{\epsilon_{1}}(t, x) u^{\epsilon_{2}}(t, x)\right)= & \int_{\mathbb{R}^{2 d}} \prod_{j=1}^{2} u_{0}\left(d \theta_{j}\right) p_{t}\left(x-\theta_{j}\right) \\
& \times \mathrm{E}\left(\exp \left(\int_{0}^{t} \Lambda_{\epsilon_{1}+\epsilon_{2}}\left(\widehat{B}_{0, t}^{1}(s)-\widehat{B}_{0, t}^{2}(s)+\frac{s\left(\theta_{1}-\theta_{2}\right)}{t}\right) d s\right)\right)
\end{aligned}
$$

converges, as $\epsilon_{1}, \epsilon_{2}$ tend to 0 , to

$$
\int_{\mathbb{R}^{2 d}} \prod_{j=1}^{2} u_{0}\left(d \theta_{j}\right) p_{t}\left(x-\theta_{j}\right) \mathrm{E}\left(\exp \left(\int_{0}^{t} \Lambda\left(\widehat{B}_{0, t}^{1}(s)-\widehat{B}_{0, t}^{2}(s)+\frac{s\left(\theta_{1}-\theta_{2}\right)}{t}\right) d s\right)\right)
$$

thanks to Proposition 3.1. Therefore, this shows the convergence of $u^{\epsilon}(t, x)$ in $L^{2}(\Omega)$ as $\epsilon \rightarrow 0$ to some limit $v(t, x)$. The fact that the convergence is in $L^{p}(\Omega)$ follows from (3.11) and Proposition 3.1 (i). Taking the limit in (3.11) as $\epsilon$ tends to zero, and using Proposition 3.1 (iii), we obtain the Feynman-Kac formula (3.9) for the moments of $v(t, x)$.

It remains to show that $v(t, x)$ coincides with the solution to equation (1.1). By the proof of the Lemma 3.1, we know that for any random variable of the form $G=e^{W(h)-\frac{1}{2}\|h\|_{\mathcal{H}}^{2}}$ with $h \in \mathcal{H}, u^{\epsilon}$ satisfies

$$
\mathrm{E}\left(G u^{\epsilon}(t, x)\right)=\left(p_{t} * u_{0}\right)(x)+\mathrm{E}\left(\left\langle\int_{[0, t] \times \mathbb{R}^{d}} p_{t-s}(x-y) u^{\epsilon}(s, y) p_{\epsilon}(x-\star), D_{s, \star} G\right\rangle_{\mathcal{H}_{0}}\right) .
$$

Now letting $\epsilon \rightarrow 0$, we see that

$$
\mathrm{E}(G v(t, x))=\left(p_{t} * u_{0}\right)(x)+\mathrm{E}\left(\left\langle v p_{t-\bullet}(x-\star), D G\right\rangle_{\mathcal{H}}\right),
$$

which implies that the process $v$ is also a solution to the equation (1.1), and by uniqueness $v=u$.

\section{Proof Theorem 1.1}

We have that $u^{\epsilon}(t, x)$ belongs to $\mathbb{D}^{\infty}$ for any $(t, x) \in(0, \infty) \times \mathbb{R}^{d}$. Moreover, we can compute its iterated Malliavin derivative using the Feynman-Kac formula (3.6): For any integer $N \geq 1$ and for $0<r_{1}<\cdots<r_{N}<t, z_{1}, \ldots z_{N} \in \mathbb{R}^{d}$,

$$
D_{\boldsymbol{r}_{N}, \boldsymbol{z}_{N}}^{N} u^{\epsilon}(t, x)=\mathrm{E}^{B}\left(u_{0}\left(B_{t}^{x}\right) \exp \left(\int_{0}^{t} W^{\epsilon}\left(d s, B_{t-s}^{x}\right)-t(2 \pi)^{d} \Lambda_{\epsilon}(0)\right) \prod_{m=1}^{N} p_{\epsilon}\left(B_{t-r_{m}}^{x}-z_{m}\right)\right),
$$

where $B$ is a $d$-dimensional Brownian motion independent of $W$ and $B_{t}^{x}=B_{t}+x$. Set

$$
M_{k, \epsilon}=\mathrm{E}\left(\left(D_{\boldsymbol{r}_{N}, \boldsymbol{z}_{N}}^{N} u^{\epsilon}(t, x)\right)^{k}\right)
$$


and

$$
M_{k}=\mathrm{E}\left(\left(D_{\boldsymbol{r}_{N}, \boldsymbol{z}_{N}}^{N} u(t, x)\right)^{k}\right) .
$$

After calculations similar to those in (3.11), we get

$$
M_{k, \epsilon}=\mathrm{E}\left(\Psi_{t, x}^{k} \exp \left(\sum_{1 \leq j<l \leq k} \int_{0}^{t} \Lambda_{2 \epsilon}\left(B_{s}^{j, x}-B_{s}^{l, x}\right) d s\right) \prod_{j=1}^{k} \prod_{m=1}^{N} p_{\epsilon}\left(B_{t-r_{m}}^{j, x}-z_{m}\right)\right),
$$

where $\Psi_{t, x}^{k}=: \prod_{j=1}^{k} u_{0}\left(B_{t}^{j}+x\right)$ and the $B^{j}, j=1 \ldots, k$ are independent $d$-dimensional Brownian motions. The $k(N+1) d$-dimensional random vector $\left(B_{t-r_{N}}^{j}+x, \ldots, B_{t-r_{1}}^{j}+x, B_{t}^{j}+x\right)_{1 \leq j \leq k}$ has the probability density

$$
\varphi(\boldsymbol{\eta}, \boldsymbol{\theta})=\prod_{j=1}^{k} p_{r_{1}}\left(\eta_{1}^{j}-\theta^{j}\right)\left(\prod_{m=1}^{N-1} p_{r_{m+1}-r_{m}}\left(\eta_{m+1}^{j}-\eta_{m}^{j}\right)\right) p_{t-r_{N}}\left(x-\eta_{N}^{j}\right),
$$

with the convention $r_{N+1}=t, r_{0}=0$. In the above expression, $\eta_{m}^{j}$ denotes the value of the random variable $B_{t-m}^{j}+x$ for $j=1, \ldots, k$ and $m=1, \ldots, N$ and $\theta^{j}$ denotes the value of $B_{t}^{j}+x$ for $j=1, \ldots, k$ and we make use of the notation $\boldsymbol{\eta}=\left(\eta_{m}^{j}\right)_{1 \leq j \leq k, 1 \leq m \leq N} \in \mathbb{R}^{k(N+1) d}$ and $\boldsymbol{\theta}=\left(\theta^{1}, \ldots, \theta^{k}\right) \in \mathbb{R}^{k d}$.

Set $\boldsymbol{\eta}_{N}^{j}=\left(\eta_{N}^{j}, \ldots, \eta_{1}^{j}\right)$ and $\boldsymbol{t}-\boldsymbol{r}_{N}=\left(t-r_{N}, \ldots, t-r_{1}\right)$. Let

$$
\left\{\widehat{B}_{0, t-\boldsymbol{r}_{N}, t}^{j, x, \boldsymbol{\eta}^{j}, \theta^{j}}(s), s \in[0, t]\right\}, \quad j=1, \ldots, k
$$

be a family of $k$ independent $d$-dimensional Brownian motions, starting at $x$ and pinned at times $t-r_{N}<\cdots<t-r_{1}<t$ to be equal to $\eta_{N}^{j}, \ldots, \eta_{1}^{j}, \theta^{j}$, respectively.

Now, conditioning on $B_{t-r_{m}}^{j}+x=\eta_{m}^{j}$ and $B_{t}^{j}+x=\theta^{j}$ for $m=1, \ldots, N$ and $j=1, \ldots, k$, we can write the expectation in equation (4.1) as follows:

$$
M_{k, \epsilon}=\int_{\mathbb{R}^{(N+1) k d}} H_{\epsilon}^{t, \boldsymbol{r}_{N}}(\boldsymbol{\eta}, \boldsymbol{\theta})\left(\prod_{j=1}^{k} \prod_{m=1}^{N} p_{\epsilon}\left(\eta_{m}^{j}-z_{m}\right)\right) \varphi(\boldsymbol{\eta}, \boldsymbol{\theta}) u_{0}(d \boldsymbol{\theta}) d \boldsymbol{\eta},
$$

where

$$
H_{\epsilon}^{t, \boldsymbol{r}_{N}}(\boldsymbol{\eta}, \boldsymbol{\theta}):=\mathrm{E}\left(\exp \left(\sum_{1 \leq j<l \leq k} \int_{0}^{t} \Lambda_{2 \epsilon}\left(\widehat{B}_{0, t-\boldsymbol{r}_{N}, t}^{j, x, \eta^{j}, \theta^{j}}(s)-\widehat{B}_{0, \boldsymbol{t}-\boldsymbol{r}_{N}, t}^{l, x, \boldsymbol{\eta}_{N}^{l}, \theta^{l}}(s)\right) d s\right)\right)
$$

and $u_{0}(d \boldsymbol{\theta})=\prod_{j=1}^{k} u_{0}\left(d \theta^{j}\right)$.

Proposition 4.1. Suppose $\Lambda$ satisfies the Dalang's condition (2.2) and $k \geq 2$ be fixed. With the above notation let $\left\{\widehat{B}_{0, \boldsymbol{t}-\boldsymbol{r}_{N}, t}^{j, x}, \boldsymbol{\eta}_{N}^{j}, \theta^{j}(s), s \in[0, t]\right\}, j=1, \ldots, k$ be a family of $k$ independent $d$ dimensional Brownian motions, starting at $x$ and pinned at times $t-r_{N}<\cdots<t-r_{1}<t$ to be equal to $\eta_{N}^{j}, \ldots, \eta_{1}^{j}, \theta^{j}$. For $\kappa \in \mathbb{R}$ we set

$$
H_{\kappa, \epsilon}^{t, \boldsymbol{r}_{N}}(\boldsymbol{\eta}, \boldsymbol{\theta})=: \mathrm{E}\left(\exp \left(\kappa \sum_{1 \leq j<l \leq k} \int_{0}^{t} \Lambda_{2 \epsilon}\left(\widehat{B}_{0, \boldsymbol{t}-\boldsymbol{r}_{N}, t}^{j, x, \boldsymbol{\eta}_{N}^{j}, \theta^{j}}(s)-\widehat{B}_{0, \boldsymbol{t}-\boldsymbol{r}_{N}, t}^{l, x, \boldsymbol{\eta}^{l}, \theta^{l}}(s)\right) d s\right)\right) .
$$

Then,

$$
\sup _{\epsilon>0} \sup _{0<r_{1}<\cdots<r_{N}<t} \sup _{(\boldsymbol{\eta}, \boldsymbol{\theta}) \in \mathbb{R}^{k(N+1) d}} H_{\kappa, \epsilon}^{t, \boldsymbol{r}_{N}}(\boldsymbol{\eta}, \boldsymbol{\theta})<\infty
$$

and $H_{\kappa, \epsilon}^{t, \boldsymbol{r}_{N}}(\boldsymbol{\eta}, \boldsymbol{\theta})$ converges to

$$
H_{\kappa}^{t, \boldsymbol{r}_{N}}(\boldsymbol{\eta}, \boldsymbol{\theta})=: \mathrm{E}\left(\exp \left(\kappa \sum_{1 \leq j<l \leq k} \int_{0}^{t} \Lambda\left(\left(\widehat{B}_{0, \boldsymbol{t}-\boldsymbol{r}_{N}, t}^{j, x, \boldsymbol{\eta}_{N}^{j}, \theta^{j}}(s)-\widehat{B}_{0, \boldsymbol{t}-\boldsymbol{r}_{N}, t}^{l, x, \boldsymbol{\eta}^{l}, \theta^{l}}(s)\right) d s\right)\right),\right.
$$

as $\epsilon \rightarrow 0$ uniformly in $\boldsymbol{\eta}, \boldsymbol{\theta}$ and $\boldsymbol{r}_{N}$. 
Proof. Consider the decomposition

$\left.\int_{0}^{t} \Lambda_{2 \epsilon}\left(\widehat{B}_{0, \boldsymbol{t}-\boldsymbol{r}_{N}, t}^{j, x, \boldsymbol{\eta}_{N}^{j}, \theta^{j}}(s)-\widehat{B}_{0, \boldsymbol{t}-\boldsymbol{r}_{N}, t}^{l, x,,_{N}^{l}, \theta^{l}}(s)\right)(s)\right) d s=\sum_{m=0}^{N} \int_{t-r_{m+1}}^{t-r_{m}} \Lambda_{2 \epsilon}\left(\widehat{B}_{0, \boldsymbol{t}-\boldsymbol{r}_{N}, t}^{j, x, \boldsymbol{\eta}_{N}^{j}, \theta^{j}}(s)-\widehat{B}_{0, \boldsymbol{t}-\boldsymbol{r}_{N}, t}^{l, x, \boldsymbol{\eta}_{N}^{l}, \theta^{l}}(s)\right) d s$,

where $r_{0}=0$ and $r_{N+1}=t$. For all $j=1, \ldots, k$, and $m=0,1, \ldots, N$,

$$
\left\{\widehat{B}_{0, \boldsymbol{t}-\boldsymbol{r}_{N}, t}^{j, x, \theta^{j}, \theta^{j}}(s), s \in\left[t-r_{m+1}, t-r_{m}\right]\right\}
$$

is a Brownian bridge that starts at $\eta_{m+1}^{j}$ and ends at $\eta_{m}^{j}$ with the convention $\eta_{N+1}^{j}=x$ and $\eta_{0}^{j}=\theta^{j}$. Therefore, using (2.7), for each $j=1, \ldots, k$, and $m=0,1, \ldots, N$ we can write, for $s \in\left[t-r_{m+1}, t-r_{m}\right]$,

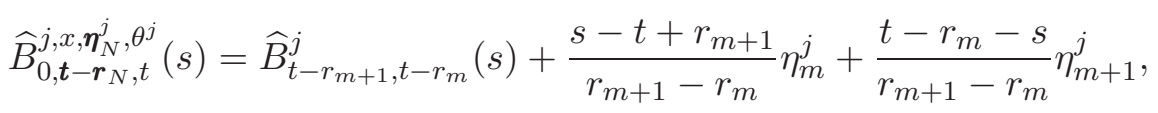

where the $\widehat{B}_{t-r_{m}, t-r_{m+1}}^{j}$ are independent $d$-dimensional Brownian bridges from 0 to 0 in each interval $\left[t-r_{m+1}, t-r_{m}\right]$. Moreover, the family of processes

$$
\left\{\widehat{B}_{t-r_{m+1}, t-r_{m}}^{j}, 1 \leq j \leq k, 0 \leq m \leq N\right\}
$$

are independent. For $j, l=1, \ldots, k$, and $m=0,1, \ldots, N-1$ set

$$
\alpha_{m}^{j, l}(s)=\frac{s-r_{m}}{r_{m+1}-r_{m}}\left(\eta_{m}^{j}-\eta_{m}^{l}\right)+\frac{r_{m+1}-s}{r_{m+1}-r_{m}}\left(\eta_{m+1}^{j}-\eta_{m+1}^{l}\right)
$$

and $\alpha_{N}^{j, l}=\frac{s-r_{N}}{t-r_{N}}\left(\eta_{N}^{j}-\eta_{N}^{l}\right)$. Then,

$$
H_{\kappa, \epsilon}^{t, \boldsymbol{r}_{N}}(\boldsymbol{\eta}, \boldsymbol{\theta})=\prod_{m=0}^{N} F_{\kappa, \epsilon}^{m}(\boldsymbol{\eta}, \boldsymbol{\theta})
$$

where for $m=0, \ldots, N$,

$$
F_{\kappa, \epsilon}^{m}(\boldsymbol{\eta}, \boldsymbol{\theta}):=\mathrm{E}\left(\exp \left(\kappa \sum_{1 \leq j<l \leq k} \int_{t-r_{m+1}}^{t-r_{m}} \Lambda_{2 \epsilon}\left(B_{t-r_{m+1}, t-r_{m}}^{j}(s)-B_{t-r_{m+1}, t-r_{m}}^{l}(s)+\alpha_{m}^{j l}(s)\right) d s\right)\right) .
$$

Then by Proposition 3.1 (i) and (iii), we have that for each fixed $t>0$ and $k \geq 2$,

$$
\sup _{\epsilon>0} \sup _{0<r_{1}<\cdots<r_{N}<t} \sup _{(\boldsymbol{\eta}, \boldsymbol{\theta}) \in \mathbb{R}^{k(N+1) d}} F_{\kappa, \epsilon}^{m}(\boldsymbol{\eta}, \boldsymbol{\theta})<\infty,
$$

and as $\epsilon \rightarrow 0, F_{\kappa, \epsilon}^{m}$ converges uniformly in $\boldsymbol{\eta}, \boldsymbol{\theta}$ and $\boldsymbol{r}_{N}$ to

$F_{\kappa}^{m}(\boldsymbol{\eta}, \boldsymbol{\theta}):=\mathrm{E}\left(\exp \left(\kappa \sum_{1 \leq j<l \leq k} \int_{t-r_{m+1}}^{t-r_{m}} \Lambda\left(B_{t-r_{m+1}, t-r_{m}}^{j}(s)-B_{t-r_{m+1}, t-r_{m}}^{l}(s)+\alpha_{m}^{j l}(s)\right) d s\right)\right)$.

The proposition follows.

Proposition 4.1 together with the expression for the moments in (4.3) imply that the family of random variables $\left\{D_{\boldsymbol{r}_{N}, \boldsymbol{z}_{N}}^{N} u^{\epsilon}(t, x), \epsilon \in(0,1]\right\}$ has uniformly bounded moments of all orders. The next result provides the limit as $\epsilon$ tends to zero of the moment of order $k$ of the iterated derivative of $u^{\epsilon}(t, x)$.

Proposition 4.2. Let $u^{\epsilon}$ be as defined in (3.6). Then for $k \geq 2$, we have, with the notation introduced in Proposition 4.1.

$$
\begin{aligned}
& \lim _{\epsilon \rightarrow 0} \mathrm{E}\left(\left(D_{\boldsymbol{r}_{N}, \boldsymbol{z}_{N}} u^{\epsilon}(t, x)\right)^{k}\right)=\left[\prod_{m=1}^{N-1} p_{r_{m+1}-r_{m}}\left(z_{m+1}-z_{m}\right)\right]^{k} p_{t-r_{N}}^{k}\left(x-z_{N}\right) \\
& \quad \times \int_{\mathbb{R}^{k d}} \prod_{j=1}^{k} u_{0}\left(d \theta^{j}\right) \prod_{j=1}^{k} p_{r_{1}}\left(z_{1}-\theta^{j}\right) \mathrm{E}\left(\exp \left(\sum_{1 \leq j<l \leq k} \int_{0}^{t} \Lambda\left(\widehat{B}_{0, t-\boldsymbol{r}_{N}, t}^{j, x, \boldsymbol{z}_{N}, \theta^{j}}(s)-\widehat{B}_{0, t-\boldsymbol{r}_{N}, t}^{l, x, \boldsymbol{z}_{N}, \theta^{l}}(s)\right) d s\right)\right) .
\end{aligned}
$$


Proof. Notice first that, by Proposition 3.1, for fixed $z_{N}$ the expression

$$
H^{t, z_{N}}(\boldsymbol{\theta}):=\mathrm{E}\left(\exp \left(\sum_{1 \leq j<l \leq k} \int_{0}^{t} \Lambda\left(\widehat{B}_{0, \boldsymbol{t}-\boldsymbol{r}_{N}, t}^{j, x, \boldsymbol{z}_{N}, \theta^{j}}(s)-\widehat{B}_{0, \boldsymbol{t}-\boldsymbol{r}_{N}, t}^{l, x, \boldsymbol{z}_{N}, \theta^{l}}(s)\right) d s\right)\right)
$$

is a bounded function of the variable $\boldsymbol{\theta}$. Thus, the integral in the above expression is well defined in view of condition (1.2). From (4.3), we obtain

$$
M_{k, \epsilon}=M_{k, \epsilon}^{(1)}+M_{k, \epsilon}^{(2)},
$$

where

$$
M_{k, \epsilon}^{(1)}:=\int_{\mathbb{R}^{(N+1) k d}}\left(H_{\epsilon}^{t, \boldsymbol{r}_{N}}(\boldsymbol{\eta}, \boldsymbol{\theta})-H^{t, \boldsymbol{r}_{N}}(\boldsymbol{\eta}, \boldsymbol{\theta})\right)\left(\prod_{j=1}^{k} \prod_{m=1}^{N} p_{\epsilon}\left(\eta_{m}^{j}-z_{m}\right)\right) \varphi(\boldsymbol{\eta}, \boldsymbol{\theta}) u_{0}(d \boldsymbol{\theta}) d \boldsymbol{\eta}
$$

and

$$
M_{k, \epsilon}^{(2)}:=\int_{\mathbb{R}^{(N+1) k d}} H^{t, \boldsymbol{r}_{N}}(\boldsymbol{\eta}, \boldsymbol{\theta})\left(\prod_{j=1}^{k} \prod_{m=1}^{N} p_{\epsilon}\left(\eta_{m}^{j}-z_{m}\right)\right) \varphi(\boldsymbol{\eta}, \boldsymbol{\theta}) u_{0}(d \boldsymbol{\theta}) d \boldsymbol{\eta},
$$

with $H_{\epsilon}^{t, \boldsymbol{r}_{N}}(\boldsymbol{\eta}, \boldsymbol{\theta})$ defined in (4.4) and

$$
H^{t, \boldsymbol{r}_{N}}(\boldsymbol{\eta}, \boldsymbol{\theta}):=E\left(\exp \left(\sum_{1 \leq j<l \leq k} \int_{0}^{t} \Lambda_{2 \epsilon}\left(\widehat{B}_{0, t-\boldsymbol{r}_{N}, t}^{j, x, \boldsymbol{\eta}_{N}^{j}, \theta^{j}}(s)-\widehat{B}_{0, \boldsymbol{t}-\boldsymbol{r}_{N}, t}^{l, x, \boldsymbol{\eta}_{N}^{l}, \theta^{l}}(s)\right) d s\right)\right) .
$$

Convergence of $M_{k, \epsilon}^{(2)}$ : We claim that

$$
\begin{aligned}
\lim _{\epsilon \rightarrow 0} M_{k, \epsilon}^{(2)}= & {\left[\prod_{m=1}^{N-1} p_{r_{m+1}-r_{m}}\left(z_{m+1}-z_{m}\right)\right]^{k} p_{t-r_{N}}^{k}\left(x-z_{N}\right) } \\
& \times \int_{\mathbb{R}^{k d}}\left(\prod_{j=1}^{k} p_{r_{1}}\left(z_{1}-\theta^{j}\right)\right) H^{t, \boldsymbol{z}_{N}}(\boldsymbol{\theta}) u_{0}(d \boldsymbol{\theta}) .
\end{aligned}
$$

Indeed, $M_{k, \epsilon}^{(2)}=\left(G * \phi_{\epsilon}\right)\left(\boldsymbol{z}_{N}, \ldots, \boldsymbol{z}_{N}\right)$, where

$$
G(\boldsymbol{\eta})=\int_{\mathbb{R}^{k d}} H^{t, \boldsymbol{r}_{N}}(\boldsymbol{\eta}, \boldsymbol{\theta})\left(\prod_{j=1}^{k} p_{r_{1}}\left(\eta_{1}^{j}-\theta^{j}\right)\right)\left(\prod_{m=1}^{N} p_{r_{m+1}-r_{m}}\left(\eta_{m+1}^{j}-\eta_{m}^{j}\right)\right) u_{0}(d \boldsymbol{\theta})
$$

with $\eta_{N+1}^{j}=x$ and

$$
\phi_{\epsilon}(\boldsymbol{\eta})=\prod_{j=1}^{k} \prod_{m=1}^{N} p_{\epsilon}\left(\eta_{m}^{j}\right) .
$$

Notice first that $G$ is integrable. In fact, taking into account that $H^{t, \boldsymbol{r}_{N}}(\boldsymbol{\eta}, \boldsymbol{\theta})$ is uniformly bounded by Proposition 4.1, we can write

$$
\begin{aligned}
\int_{\mathbb{R}^{k N d}}|G(\boldsymbol{\eta})| d \boldsymbol{\eta} & \leq C \int_{\mathbb{R}^{k(N+1) d}} \prod_{j=1}^{k} p_{r_{1}}\left(\eta_{1}^{j}-\theta^{j}\right)\left(\prod_{m=1}^{N} p_{r_{m+1}-r_{m}}\left(\eta_{m+1}^{j}-\eta_{m}^{j}\right)\right)\left|u_{0}\right|(d \boldsymbol{\theta}) d \boldsymbol{\eta} \\
& \leq C\left(\int_{\mathbb{R}^{d}}\left|u_{0}\right|(d \theta) p_{t}(x-\theta)\right)^{k}<\infty .
\end{aligned}
$$

Moreover, again by Proposition 4.1, one can show that $G$ is uniformly continuous in $\boldsymbol{\eta}_{N}^{1}, \ldots, \boldsymbol{\eta}_{N}^{k}$. Taking into account that $\phi_{\epsilon}$ is an approximation to identity in $\mathbb{R}^{N k d}$, we obtain that $\left(G * \phi_{\epsilon}\right)\left(\boldsymbol{z}_{N}, \ldots \boldsymbol{z}_{N}\right)$ converges to $G\left(\boldsymbol{z}_{N}, \ldots, \boldsymbol{z}_{N}\right)$ as $\epsilon \rightarrow 0$. So, it only remains to show that the first term $M_{k, \epsilon}^{(1)}$ converges to 0 as $\epsilon$ goes to 0 . This follows from Proposition 4.1 combined with the dominated convergence theorem. 
In the next proposition we show that the approximated Malliavin derivatives converge in $L^{p}(\Omega)$, for each $p \geq 2$.

Proposition 4.3. For all $0<r_{1}, \ldots, r_{N}<t$ and $z_{1}, \ldots, z_{N} \in \mathbb{R}^{d}$,

$$
\lim _{\epsilon \rightarrow 0} D_{\boldsymbol{r}_{N}, \boldsymbol{z}_{N}}^{N} u^{\epsilon}(t, x)
$$

exists in $L^{p}(\Omega)$, for all $p \geq 2$. We denote this limit by $\Phi_{\boldsymbol{r}_{N}, \boldsymbol{z}_{N}}(t, x)$.

Proof. First we show that $D_{\boldsymbol{r}_{N}, \boldsymbol{z}_{N}} u^{\epsilon}(t, x)$ converges in $L^{2}(\Omega)$ as $\epsilon$ tends to zero. For $\epsilon_{1}, \epsilon_{2}>0$, by calculations similar to (4.1) and denoting by $B^{1}$ and $B^{2}$ two independent $d$-dimensional Brownian motions, we have

$$
\begin{aligned}
& \mathrm{E}\left(D_{\boldsymbol{r}_{N}, \boldsymbol{z}_{N}}^{N} u^{\epsilon_{1}}(t, x) D_{\boldsymbol{r}_{N}, \boldsymbol{z}_{N}}^{N} u^{\epsilon_{2}}(t, x)\right) \\
& =\mathrm{E}\left(\exp \left(\int_{0}^{t} \Lambda_{\epsilon_{1}+\epsilon_{2}}\left(B_{s}^{1}-B_{s}^{2}\right) d s\right) \prod_{m=1}^{N} p_{\epsilon_{1}}\left(B_{t-r_{m}}^{1, x}-z_{m}\right) p_{\epsilon_{2}}\left(B_{t-r_{m}}^{2, x}-z_{m}\right)\right) \\
& =\int_{\mathbb{R}^{2(N+1) d}} u_{0}\left(d \theta^{1}\right) u_{0}\left(d \theta^{2}\right) \mathrm{E}\left(\exp \left(\int_{0}^{t} \Lambda_{\epsilon_{1}+\epsilon_{2}}\left(\widehat{B}_{0, \boldsymbol{t}-\boldsymbol{r}_{N}, t}^{1, x, \boldsymbol{\eta}_{N}^{1}, \theta^{1}}(s)-\widehat{B}_{0, \boldsymbol{t}-\boldsymbol{r}_{N}}^{2, x, \boldsymbol{\eta}_{N}^{2}, \theta^{2}}, t(s)\right) d s\right)\right) \\
& \quad \times \prod_{j=1}^{2} \prod_{m=1}^{N} p_{\epsilon_{j}}\left(\eta_{m}^{j}-z_{m}\right) \varphi(\boldsymbol{\eta}, \boldsymbol{\theta}) d \boldsymbol{\eta} .
\end{aligned}
$$

Following the same proof as in (4.2) we obtain that $\mathrm{E}\left(D_{\boldsymbol{r}_{N}, z_{N}} u^{\epsilon_{1}}(t, x) D_{\boldsymbol{r}_{N}, \boldsymbol{z}_{N}} u^{\epsilon_{2}}(t, x)\right)$ converges, as $\epsilon_{1}, \epsilon_{2} \rightarrow 0$, to

$$
\begin{aligned}
& {\left[\prod_{m=1}^{N-1} p_{r_{m+1}-r_{m}}\left(z_{m+1}-z_{m}\right)\right]^{2} p_{t-r_{N}}^{2}\left(x-z_{N}\right)} \\
& \quad \times \int_{\mathbb{R}^{2 d}} u_{0}\left(d \theta^{1}\right) u_{0}\left(d \theta^{2}\right) \prod_{j=1}^{2} p_{r_{1}}\left(z_{1}-\theta^{j}\right) \mathrm{E}\left(\exp \left(\int_{0}^{t} \Lambda\left(\widehat{B}_{0, \boldsymbol{t}-\boldsymbol{r}_{N}, t}^{1, x, \boldsymbol{z}_{N}, \theta^{1}}(s)-\widehat{B}_{0, \boldsymbol{t}-\boldsymbol{r}_{N}, t}^{2, x, \boldsymbol{z}_{N}, \theta^{2}}(s)\right) d s\right)\right) .
\end{aligned}
$$

This implies the convergence in $L^{2}(\Omega)$. By the boundedness of all moments of all orders, the convergence is in $L^{p}(\Omega)$ for all $p \geq 2$.

The next step in the proof of Theorem 1.1 is to show that the limit random field $\Phi_{\boldsymbol{r}_{N}, \boldsymbol{z}_{N}}(t, x)$ appearing in Proposition 4.3 is precisely the iterated derivative $D_{\boldsymbol{r}_{N}, \boldsymbol{z}_{N}}^{N} u(t, x)$. We recall that the iterated derivative $D^{N} u(t, x)$ is an $\mathcal{H}^{\otimes N}$-valued random variable. In the next result we will show first that $D^{N} u^{\epsilon}(t, x)$ converges to $D^{N} u(t, x)$ in $L^{2}\left(\Omega ; \mathcal{H}^{\otimes N}\right)$, as $N$ tends to infinity. The proof of this fact will be based on the Wiener chaos expansion of the solution $u(t, x)$ that we recall here. The following chaos expansion was shown in [11],

$$
u(t, x)=\left(p_{t} * u_{0}\right)(x)+\sum_{n=1}^{\infty} I_{n}\left(f_{n, t, x}\right)
$$

where $I_{n}$ denotes the multiple stochastic integral with respect to the noise $W$ and $f_{n, t, x} \in \mathcal{H}^{\otimes n}$ is the symmetric kernel given by

$$
f_{n, t, x}(s, y):=f_{n, t, x}\left(s_{1}, y_{1}, \ldots, s_{n}, y_{n}\right)=\frac{1}{n !}\left(p_{s_{\sigma(1)}} * u_{0}\right)\left(x_{\sigma(1)}\right) \prod_{i=1}^{n} p_{s_{\sigma(i+1)}-s_{\sigma(i)}}\left(y_{\sigma(i+1)}-y_{\sigma(i)}\right)
$$

for $\left(s_{1}, y_{1}, \ldots, s_{n}, y_{n}\right) \in\left((0, t) \times \mathbb{R}^{d}\right)^{n}$ satisfying $s_{i} \neq s_{j}$ for $i \neq j$. In this expression, $\sigma$ denotes the permutation of $\{1, \ldots, n\}$ such that $0<s_{\sigma(1)}<\cdots<s_{\sigma(n)}<t$ and we use the convention

\footnotetext{
(1.2)

${ }^{1}$ In $[11$ the initial condition is continuous and bounded, but the result still holds for initial conditions satisfying
} 
$\sigma(n+1)=t$ and $y_{\sigma(n+1)}=x$. In the same way, we can derive the Wiener chaos expansion of $u^{\epsilon}(t, x)$ with respect to the noise $W$ :

$$
u^{\epsilon}(t, x)=\left(p_{t} * u_{0}\right)(x)+\sum_{n=1}^{\infty} I_{n}\left(f_{n, t, x}^{\epsilon}\right)
$$

where

$$
\begin{aligned}
f_{n, t, x}^{\epsilon}(s, y):= & f_{n, t, x}^{\epsilon}\left(s_{1}, y_{1}, \ldots, s_{n}, y_{n}\right)=\frac{1}{n !} \\
& \times \int_{\mathbb{R}^{n d}}\left(p_{s_{\sigma(1)}} * u_{0}\right)\left(w_{1}\right) \prod_{i=1}^{n} p_{s_{\sigma(i+1)}-s_{\sigma(i)}}\left(w_{i+1}-w_{i}\right) p_{\epsilon}\left(y_{\sigma(i)}-w_{i}\right) d w_{1} \cdots d w_{n},
\end{aligned}
$$

with the same convention about $\sigma$ mentioned above.

Proposition 4.4. $\lim _{\epsilon \rightarrow 0} D^{N} u^{\epsilon}(t, x)=D^{N} u(t, x)$ in $L^{2}\left(\Omega ; \mathcal{H}^{\otimes N}\right)$.

Proof. Define

$$
g_{n, t, x}^{\epsilon}(s, y):=f_{n, t, x}^{\epsilon}(s, y)-f_{n, t, x}(s, y) .
$$

We know that, in terms of the Wiener chaos expansion, the Malliavin derivative is obtained by leaving one variable free and multiplying by the order of the chaos. That is,

$$
D_{\boldsymbol{r}_{N}, \boldsymbol{z}_{N}}^{N}\left(u^{\epsilon}(t, x)-u(t, x)\right)=\sum_{n=N}^{\infty} n(n-1) \ldots(n-N+1) I_{n-N}\left(\left(g_{n, t, x}^{\epsilon}\left(\bullet, r_{1}, z_{1}, \ldots, r_{N}, z_{N}\right)\right)\right),
$$

for any $z_{1}, \ldots, z_{N} \in \mathbb{R}^{d}$ and $0<r_{1}<\cdots<r_{N}$ and with the notation $\boldsymbol{r}_{N}=\left(r_{1}, \ldots, r_{N}\right)$ and $z_{N}=\left(z_{1}, \ldots, z_{N}\right)$. This leads to

$$
\mathrm{E}\left(\left|D_{\boldsymbol{r}_{N}, \boldsymbol{z}_{N}}^{N}\left(u^{\epsilon}(t, x)-u(t, x)\right)\right|^{2}\right) \leq \sum_{n=N}^{\infty} n^{N} n !\left\|g_{n, t, x}^{\epsilon}\left(\bullet, r_{1}, z_{1}, \ldots, r_{N}, z_{N}\right)\right\|_{\mathcal{H}^{\otimes(n-N)}}^{2},
$$

which implies

$$
\mathrm{E}\left(\left\|D^{N}\left(u^{\epsilon}(t, x)-u(t, x)\right)\right\|_{\mathcal{H}^{\otimes N}}^{2}\right) \leq \sum_{n=N}^{\infty} n^{N} n !\left\|g_{n, t, x}^{\epsilon}\right\|_{\mathcal{H}^{\otimes n}}^{2} .
$$

Using the notation $\boldsymbol{\xi}=\left(\xi_{1}, \ldots, \xi_{n}\right), \mu(d \boldsymbol{\xi})=\prod_{i=1}^{n} \mu\left(d \xi_{1}\right)$ and $d \boldsymbol{s}=\prod_{i=1}^{n} d s_{i}$, we have

$$
\left\|g_{n, t, x}^{\epsilon}\right\|_{\mathcal{H}^{\otimes n}}^{2}=\int_{[0, t]^{n}} \int_{\mathbb{R}^{n d}}\left|\mathscr{F} g_{n, t, x}^{\epsilon}(\xi)\right|^{2} \mu(d \boldsymbol{\xi}) d \boldsymbol{s},
$$

where $\mathscr{F}$ denotes the Fourier transform in the space variables. Now let us calculate the Fourier transform of $g_{n, t, x}^{\epsilon}$ :

$$
\left|\mathscr{F} g_{n, t, x}^{\epsilon}(\boldsymbol{\xi})\right|^{2}=\frac{1}{(n !)^{2}}\left(1-e^{-\epsilon \sum_{i=1}^{n}\left|\xi_{i}\right|^{2}}\right) \prod_{i=1}^{n} \exp \left(-\left(s_{\sigma(i+1)}-s_{\sigma(i)}\right)\left|\sum_{j=1}^{i} \xi_{\sigma(j)}\right|^{2}\right),
$$

which tends to zero for all $\xi \in \mathbb{R}^{n d}$ and for all $n \geq 1$, as $\epsilon \rightarrow 0$.

By the dominated convergence theorem, in order to show that $\lim _{\epsilon \rightarrow 0} D^{N} u^{\epsilon}(t, x)=D^{N} u(t, x)$ in $L^{2}\left(\Omega ; \mathcal{H}^{\otimes N}\right)$, it suffices to check that

$$
I:=\sum_{n=1}^{\infty} \frac{n^{N}}{n !} \int_{[0, t]^{n}} \int_{\mathbb{R}^{n d}} \prod_{i=1}^{n} \exp \left(-\left(s_{\sigma(i+1)}-s_{\sigma(i)}\right)\left|\sum_{j=1}^{i} \xi_{\sigma(j)}\right|^{2}\right) \mu(d \boldsymbol{\xi}) d \boldsymbol{s}<\infty .
$$

We can write

$$
I=\sum_{n=1}^{\infty} n^{N} \int_{[0, t]_{<}^{n}} \int_{\mathbb{R}^{n d}} \prod_{i=1}^{n} \exp \left(-\left(s_{i+1}-s_{i}\right)\left|\xi_{1}+\cdots \xi_{j}\right|^{2}\right) \mu(d \boldsymbol{\xi}) d \boldsymbol{s},
$$


where we used the notation $[0, t]_{<}^{n}:=\left\{\left(s_{1}, \ldots, s_{n}\right): 0<s_{1}<\cdots<s_{n}<t\right\}$ and the convention $s_{0}=0$. This leads to the estimate

$$
\begin{aligned}
I & \leq \sum_{n=1}^{\infty} n^{N} \int_{[0, t]_{<}^{n}} \prod_{j=1}^{n}\left(\sup _{\eta \in \mathbb{R}^{d}} \int_{\mathbb{R}^{d}} \exp \left(-\left(s_{j}-s_{j-1}\right)\left|\xi_{j}+\eta\right|^{2}\right) d \mu\left(\xi_{j}\right)\right) d s_{1} \cdots d s_{n} \\
& =\sum_{n=1}^{\infty} n^{N} \int_{[0, t]^{n}} \prod_{j=1}^{n}\left(\int_{\mathbb{R}^{d}} \exp \left(-\left(s_{j}-s_{j-1}\right)\left|\xi_{j}\right|^{2}\right) d \mu\left(\xi_{j}\right)\right) d s_{1} \cdots d s_{n}=: \sum_{n=1}^{\infty} n^{N} J_{n},
\end{aligned}
$$

because, as it is easy to check using the spectral measure $\mu$, the above supremum is attained at $\eta=0$. Let $w_{j}:=s_{j}-s_{j-1}, d \boldsymbol{w}=d w_{1} \cdots d w_{n}$, and $A_{t, n}:=\left\{\left(w_{1}, \ldots, w_{n}\right): w_{j} \geq 0, j=\right.$ $1, \ldots, n$ and $\left.w_{1}+\cdots+w_{n} \leq t\right\}$. Then, integral in (4.5) is equal to

$$
J_{n}=\int_{A_{t, n}} \int_{\mathbb{R}^{d n}} \exp \left(-\sum_{j=1}^{n} w_{j}\left|\xi_{j}\right|^{2}\right) d \mu(\boldsymbol{\xi}) d \boldsymbol{w} .
$$

To estimate this quantity, set

$$
C_{M}:=\int_{|\xi| \geq M} \frac{\mu(d \xi)}{|\xi|^{2}} \text {, and } D_{M}:=\mu\left(\left\{\xi \in \mathbb{R}^{d}:|\xi| \leq M\right\}\right) .
$$

By Dalang's condition (2.2), both $C_{M}, D_{M}$ are finite, and, we can choose $M>0$ such that $C_{M}<\frac{1}{4}$. Thus, by [11, Lemma 3.3], we get

$$
J_{n} \leq \sum_{k=0}^{n}\left(\begin{array}{l}
n \\
k
\end{array}\right) \frac{t^{k}}{k !} D_{M}^{k}\left(2 C_{M}\right)^{n-k}
$$

Substituting (4.6) into (4.5), we get

$$
I \leq \sum_{n=1}^{\infty} \sum_{k=0}^{n} n^{N}\left(\begin{array}{l}
n \\
k
\end{array}\right) \frac{t^{k}}{k !} D_{M}^{k}\left(2 C_{M}\right)^{n-k} \leq \sum_{k=0}^{\infty} \frac{\left(2 D_{M} t\right)^{k}}{k !} \sum_{n=k}^{\infty} n^{N}\left(4 C_{M}\right)^{n-k}<\infty,
$$

which allows us to conclude the proof.

In the next result we show that $D^{N} u(t, x)$ is actually a function and it coincides with $\left(\boldsymbol{r}_{N}, \boldsymbol{z}_{N}\right) \mapsto \Phi_{\boldsymbol{r}_{N}, \boldsymbol{z}_{N}}(t, x)$, where $\Phi_{\boldsymbol{r}_{N}, \boldsymbol{z}_{N}}(t, x)$ is the limiting random field in Proposition 4.3 .

Proposition 4.5. Let $\Phi_{\boldsymbol{r}_{N}, \boldsymbol{z}_{N}}(t, x)$ be as in Proposition 4.3. Then, $\Phi_{\boldsymbol{r}_{N}, \boldsymbol{z}_{N}}(t, x)=D_{\boldsymbol{r}_{N}, \boldsymbol{z}_{N}}^{N} u(t, x)$ for almost all $\left(\boldsymbol{r}_{N}, \boldsymbol{z}_{N}\right) \in[0, t]_{<}^{N} \times \mathbb{R}^{N d}$.

Proof. The proof will be done in two steps.

Step 1: In this step we will show that for any bounded and continuous function $\Psi:[0, t]^{N} \times$ $\mathbb{R}^{N d} \rightarrow \mathbb{R}$, the limit

$$
\lim _{\epsilon \rightarrow 0}\left\langle D^{N} u^{\epsilon}(t, x), \Psi\right\rangle_{L^{2}\left([0, t]^{N} \times \mathbb{R}^{N d}\right)}=\langle\Phi(t, x), \Psi\rangle_{L^{2}\left([0, t]^{N} \times \mathbb{R}^{N d}\right)}
$$

holds in $L^{2}(\Omega)$. We already know, by Proposition 4.3, that for all $0<r_{1}<\cdots<r_{N}<t$ and for all $z_{N} \in \mathbb{R}^{d N}$ the $\operatorname{limit}_{\lim _{\epsilon \rightarrow 0}} D_{\boldsymbol{r}_{N}, \boldsymbol{z}_{N}}^{N} u^{\epsilon}(t, x)=\Phi_{\boldsymbol{r}_{N}, \boldsymbol{z}_{N}}(t, x)$ holds in $L^{2}(\Omega)$. Then, the convergence (4.7) holds if

$$
\begin{gathered}
\lim _{\epsilon_{1}, \epsilon_{2} \rightarrow 0} \mathrm{E}\left(\int_{\left([0, t]^{N}\right)^{2}} \int_{\mathbb{R}^{2 N d}} D_{\boldsymbol{r}_{N}, \boldsymbol{z}_{N}}^{N} u^{\epsilon_{1}}(t, x) D_{\boldsymbol{s}_{N}, \boldsymbol{y}_{N}}^{N} u^{\epsilon_{2}}(t, x) \Psi\left(\boldsymbol{r}_{N}, \boldsymbol{z}_{N}\right) \Psi\left(\boldsymbol{s}_{N}, \boldsymbol{y}_{N}\right) d \boldsymbol{z}_{N} d \boldsymbol{y}_{N} d \boldsymbol{r}_{N} d \boldsymbol{s}_{N}\right) \\
=\mathrm{E}\left(\int_{\left([0, t]^{N}\right)^{2}} \int_{\mathbb{R}^{2 N d}} \Phi_{\boldsymbol{r}_{N}, \boldsymbol{z}_{N}}(t, x) \Phi_{\boldsymbol{s}_{N}, \boldsymbol{y}_{N}}(t, x) \Psi\left(\boldsymbol{r}_{N}, \boldsymbol{z}_{N}\right) \Psi\left(\boldsymbol{s}_{N}, \boldsymbol{y}_{N}\right) d \boldsymbol{z}_{N} d \boldsymbol{y}_{N} d \boldsymbol{r}_{N} d \boldsymbol{s}_{N}\right) .
\end{gathered}
$$

Set

$M_{\epsilon_{1}, \epsilon_{2}}=\mathrm{E}\left(\int_{\left([0, t]_{<}^{N}\right)^{2}} \int_{\mathbb{R}^{2 N d}} D_{\boldsymbol{r}_{N}, \boldsymbol{z}_{N}}^{N} u^{\epsilon_{1}}(t, x) D_{\boldsymbol{s}_{N}, \boldsymbol{y}_{N}}^{N} u^{\epsilon_{2}}(t, x) \Psi\left(\boldsymbol{r}_{N}, \boldsymbol{z}_{N}\right) \Psi\left(\boldsymbol{s}_{N}, \boldsymbol{y}_{N}\right) d \boldsymbol{z}_{N} d \boldsymbol{y}_{N} d \boldsymbol{r}_{N} d \boldsymbol{s}_{N}\right)$. 
We have

$$
\begin{aligned}
M_{\epsilon_{1}, \epsilon_{2}}= & \int_{\left([0, t]_{<}^{N}\right)^{2}} d \boldsymbol{r}_{N} d \boldsymbol{s}_{N} \int_{\mathbb{R}^{2 N d}} d \boldsymbol{z}_{N} d \boldsymbol{y}_{N} \Psi\left(\boldsymbol{r}_{N}, \boldsymbol{z}_{N}\right) \Psi\left(\boldsymbol{s}_{N}, \boldsymbol{y}_{N}\right) \\
& \times \int_{\mathbb{R}^{2(N+1) d}} H_{\epsilon_{1}, \epsilon_{2}}^{2, \boldsymbol{r}_{N}}(\boldsymbol{\eta}, \boldsymbol{\theta}) u_{0}\left(d \theta^{1}\right) u_{0}\left(d \theta^{2}\right)\left(\prod_{m=1}^{N} p_{\epsilon_{1}}\left(\eta_{m}^{1}-z_{m}\right) p_{\epsilon_{2}}\left(\eta_{m}^{2}-y_{m}\right)\right) \varphi(\boldsymbol{\eta}, \boldsymbol{\theta}) d \boldsymbol{\eta},
\end{aligned}
$$

where

$$
H_{\epsilon_{1}, \epsilon_{2}}^{2, \boldsymbol{r}_{N}}(\boldsymbol{\eta}, \boldsymbol{\theta})=: \mathrm{E}\left(\exp \left(\int_{0}^{t} \Lambda_{\epsilon_{1}+\epsilon_{2}}\left(\widehat{B}_{0, \boldsymbol{t}-\boldsymbol{r}_{N}, t}^{1, x, \boldsymbol{\eta}_{N}^{1}, \theta^{1}}(s)-\widehat{B}_{0, \boldsymbol{t}-\boldsymbol{r}_{N}, t}^{2, x,,_{N}^{2}, \theta^{2}}(s)\right) d s\right)\right),
$$

where we used the notation introduced in (4.2). From Proposition 3.1, we know that $H_{\epsilon_{1}, \epsilon_{2}}^{2, \boldsymbol{r}_{N}}(\boldsymbol{\eta}, \boldsymbol{\theta})$ is uniformly bounded and converges as $\epsilon_{1}$ and $\epsilon_{2}$ tend to zero, uniformly in $\boldsymbol{\eta}, \boldsymbol{\theta}$ and $\boldsymbol{r}_{N}$ to

$$
H^{2, \boldsymbol{r}_{N}}(\boldsymbol{\eta}, \boldsymbol{\theta})=: \mathrm{E}\left(\exp \left(\int_{0}^{t} \Lambda\left(\widehat{B}_{0, \boldsymbol{t}-\boldsymbol{r}_{N}, t}^{1, x, \boldsymbol{\eta}_{1}^{1}, \theta^{1}}(s)-\widehat{B}_{0, \boldsymbol{t}-\boldsymbol{r}_{N}, t}^{2, x, \boldsymbol{\eta}^{2}, \theta^{2}}(s)\right) d s\right)\right) .
$$

Moreover,

$$
\begin{aligned}
& \int_{\left([0, t]_{<}^{N}\right)^{2}} d \boldsymbol{r}_{N} d \boldsymbol{s}_{N} \int_{\mathbb{R}^{2 N d}} d \boldsymbol{z}_{N} d \boldsymbol{y}_{N}\left|\Psi\left(\boldsymbol{r}_{N}, \boldsymbol{z}_{N}\right) \Psi\left(\boldsymbol{s}_{N}, \boldsymbol{y}_{N}\right)\right| \\
& \quad \times \int_{\mathbb{R}^{2(N+1) d}}\left|u_{0}\right|\left(d \theta^{1}\right)\left|u_{0}\right|\left(d \theta^{2}\right)\left(\prod_{m=1}^{N} p_{\epsilon_{1}}\left(\eta_{m}^{1}-z_{m}\right) p_{\epsilon_{2}}\left(\eta_{m}^{2}-y_{m}\right)\right) \varphi(\boldsymbol{\eta}, \boldsymbol{\theta}) d \boldsymbol{\eta} \\
& \leq\|\Psi\|_{\infty}^{2} \frac{t^{N}}{N !}\left(\int_{\mathbb{R}^{d}}\left|u_{0}\right|(d y) p_{t}(x-y)\right)^{2}<\infty .
\end{aligned}
$$

As a consequence,

$$
\lim _{\epsilon_{1}, \epsilon_{2} \rightarrow 0} M_{\epsilon_{1}, \epsilon_{2}}=\lim _{\epsilon_{1}, \epsilon_{2} \rightarrow 0} N_{\epsilon_{1}, \epsilon_{2}}
$$

where

$$
\begin{aligned}
N_{\epsilon_{1}, \epsilon_{2}}= & \int_{\left([0, t]_{<}^{N}\right)^{2}} d \boldsymbol{r}_{N} d \boldsymbol{s}_{N} \int_{\mathbb{R}^{2 N d}} d \boldsymbol{z}_{N} d \boldsymbol{y}_{N} \Psi\left(\boldsymbol{r}_{N}, \boldsymbol{z}_{N}\right) \Psi\left(\boldsymbol{s}_{N}, \boldsymbol{y}_{N}\right) \\
& \times \int_{\mathbb{R}^{2(N+1) d}} H^{2, \boldsymbol{r}_{N}}(\boldsymbol{\eta}, \boldsymbol{\theta}) u_{0}\left(d \theta^{1}\right) u_{0}\left(d \theta^{2}\right)\left(\prod_{m=1}^{N} p_{\epsilon_{1}}\left(\eta_{m}^{1}-z_{m}\right) p_{\epsilon_{2}}\left(\eta_{m}^{2}-y_{m}\right)\right) \varphi(\boldsymbol{\eta}, \boldsymbol{\theta}) d \boldsymbol{\eta} .
\end{aligned}
$$

Finally, taking into account that $\Psi$ is continuous and bounded, we deduce the convergence

$$
\begin{aligned}
\lim _{\epsilon_{1}, \epsilon_{2} \rightarrow 0} N_{\epsilon_{1}, \epsilon_{2}}= & \int_{\left([0, t]_{<}^{N}\right)^{2}} d \boldsymbol{r}_{N} d \boldsymbol{s}_{N} \int_{\mathbb{R}^{2 N d}} d \boldsymbol{z}_{N} d \boldsymbol{y}_{N} \Psi\left(\boldsymbol{r}_{N}, \boldsymbol{z}_{N}\right) \Psi\left(\boldsymbol{s}_{N}, \boldsymbol{y}_{N}\right) \\
& \times \int_{\mathbb{R}^{2(N+1) d}} H^{2, \boldsymbol{r}_{N}}\left(\boldsymbol{z}_{N}, \boldsymbol{y}_{N}, \boldsymbol{\theta}\right) u_{0}\left(d \theta^{1}\right) u_{0}\left(d \theta^{2}\right) \varphi(\boldsymbol{\eta}, \boldsymbol{\theta}) d \boldsymbol{\eta},
\end{aligned}
$$

where

$$
H^{2, \boldsymbol{r}_{N}}\left(\boldsymbol{z}_{N}, \boldsymbol{y}_{N}, \boldsymbol{\theta}\right)=: \mathrm{E}\left(\exp \left(\int_{0}^{t} \Lambda\left(\widehat{B}_{0, \boldsymbol{t}-\boldsymbol{r}_{N}, t}^{1, x, \boldsymbol{z}_{N}, \theta^{1}}(s)-\widehat{B}_{0, \boldsymbol{t}-\boldsymbol{r}_{N}, t}^{2, x, \boldsymbol{y}_{N}, \theta^{2}}(s)\right) d s\right)\right) .
$$

This shows that

$$
\lim _{\epsilon_{1}, \epsilon_{2} \rightarrow 0} M_{\epsilon_{1}, \epsilon_{2}}=\mathrm{E}\left(\int_{\left([0, t]_{<}^{N}\right)^{2}} \int_{\mathbb{R}^{2 N d}} \Phi_{\boldsymbol{r}_{N}, \boldsymbol{z}_{N}}(t, x) \Phi_{\boldsymbol{s}_{N}, \boldsymbol{y}_{N}}(t, x) \Psi\left(\boldsymbol{r}_{N}, \boldsymbol{z}_{N}\right) \Psi\left(\boldsymbol{s}_{N}, \boldsymbol{y}_{N}\right) d \boldsymbol{z}_{N} d \boldsymbol{y}_{N} d \boldsymbol{r}_{N} d \boldsymbol{s}_{N}\right)
$$

and completes the proof of the convergence (4.7).

Step 2: We want to show that $D^{N} u(t, x)$, which is defined as a random variable taking values in $\mathcal{H}^{\otimes N}$, is actually a function and coincides with $\Phi^{N}(t, x)$, for almost all $\left(\boldsymbol{r}_{N}, \boldsymbol{z}_{N}\right) \in[0, t]_{<}^{N} \times \mathbb{R}^{N d}$, almost everywhere. Because $\mathscr{S}\left(\mathbb{R}_{+}^{N} \times \mathbb{R}^{N d}\right)$ is dense in $\mathcal{H}^{\otimes N}$, it suffices to show that for every $h \in \mathscr{S}\left(\mathbb{R}_{+}^{N} \times \mathbb{R}^{N d}\right)$, we have

$$
\left\langle D^{N} u(t, x), h\right\rangle_{\mathcal{H}^{\otimes N}}=\left\langle\Phi^{N}(t, x), h\right\rangle_{\mathcal{H}^{\otimes N}} .
$$


We know, from Proposition 4.4 above, that $\left\langle D^{N} u(t, x), h\right\rangle_{\mathcal{H}^{\otimes N}}$ is the limit in $L^{2}(\Omega)$ as $\epsilon \rightarrow 0$ of $\left\langle D^{N} u^{\epsilon}(t, x), h\right\rangle_{\mathcal{H}^{\otimes N}}$. Moreover,

$$
\left\langle D^{N} u^{\epsilon}(t, x), h\right\rangle_{\mathcal{H}^{\otimes N}}=\int_{[0, t]_{<}^{N}} \int_{\mathbb{R}^{2 N d}} D_{\boldsymbol{r}_{N}, \boldsymbol{z}_{N}}^{N} u^{\epsilon}(t, x) h\left(\boldsymbol{r}_{N}, \boldsymbol{z}_{N}-\boldsymbol{y}_{N}\right) \Lambda\left(d \boldsymbol{y}_{N}\right) d \boldsymbol{z}_{N} d \boldsymbol{r}_{N} .
$$

The function

$$
\Psi\left(\boldsymbol{r}_{N}, \boldsymbol{z}_{N}\right):=\int_{\mathbb{R}^{N d}} h\left(\boldsymbol{r}_{N}, \boldsymbol{z}_{N}-\boldsymbol{y}_{N}\right) \Lambda\left(d \boldsymbol{y}_{N}\right)
$$

is continuous and bounded because it can be written as

$$
\Psi\left(\boldsymbol{r}_{N}, \boldsymbol{z}_{N}\right)=\int_{\mathbb{R}^{N d}} e^{-i \boldsymbol{z}_{N} \cdot \boldsymbol{\xi} \mathscr{F}} h\left(\boldsymbol{r}_{N}, \boldsymbol{\xi}\right) \mu(d \boldsymbol{\xi})
$$

and $\int_{\mathbb{R}^{N d}}\left|\mathscr{F} h\left(\boldsymbol{r}_{N}, \boldsymbol{\xi}\right)\right| \mu(d \boldsymbol{\xi})<\infty$. From Step 1 applied to the function $\Psi$ defined in (4.9), we deduce

$$
\begin{aligned}
\lim _{\epsilon \rightarrow 0}\left\langle D^{N} u^{\epsilon}(t, x), h\right\rangle_{\mathcal{H} \otimes N} & =\int_{[0, t]_{<}^{N}} \int_{\mathbb{R}^{2 N d}} \Phi_{\boldsymbol{r}_{N}, \boldsymbol{r}_{N}}^{N} h\left(\boldsymbol{r}_{N}, \boldsymbol{z}_{N}-\boldsymbol{y}_{N}\right) \Lambda\left(d \boldsymbol{y}_{N}\right) d \boldsymbol{z}_{N} d \boldsymbol{r}_{N} \\
& =\left\langle\Phi^{N}(t, x), h\right\rangle_{\mathcal{H} \otimes N},
\end{aligned}
$$

where the convergence is in $L^{2}(\Omega)$. This completes the proof of the proposition.

Proof of Theorem [1.1. From Proposition 4.5, iterated Malliavin derivative $D_{\boldsymbol{r}_{N}, \boldsymbol{z}_{N}}^{N} u(t, x)$ coincides with the random field $\Phi_{\boldsymbol{r}_{N}, \boldsymbol{z}_{N}}(t, x)$ for almost all $\boldsymbol{r}_{N}$ and $\boldsymbol{z}_{N}$. Therefore, using Proposition 4.3 the moment of order $k$ of $D_{\boldsymbol{r}_{N}, \boldsymbol{z}_{N}}^{N_{N}} u(t, x)$ will be the limit as $\epsilon \rightarrow 0$ of the moment of order $k$ of $D_{\boldsymbol{r}_{N}, \boldsymbol{z}_{N}}^{N} u^{\epsilon}(t, x)$, which has been computed in Proposition 4.2

\section{REFERENCES}

[1] L. Chen R. C. Dalang: Moments, intermittency, and growth indices for the nonlinear stochastic heat equation with rough initial conditions. Ann. Probab. 43 (2015), no. 6, 3006-3051.

[2] L. Chen and R. C. Dalang: Hölder-continuity for the nonlinear stochastic heat equation with rough initial conditions. Stoch. Partial Differ. Equ. Anal. Comput. 2 (2014), no. 3, 316-352.

[3] L. Chen, D. Khoshnevisan, D. Nualart, and F. Pu: Spatial ergodicity for SPDEs via Poincaré-type inequalities. Preprint, 2019.

[4] L. Chen, D. Khoshnevisan, D. Nualart, and F. Pu: Poincaré inequality, and central limit theorems for parabolic stochastic partial differential equations. Preprint, 2019.

[5] L. Chen, D. Khoshnevisan, D. Nualart, and F. Pu: Spatial ergodicity and central limit theorems for parabolic Anderson model with delta initial condition. In preparation, 2020.

[6] L. Chen, D. Khoshnevisan, D. Nualart, and F. Pu: Central limit theorems for spatial averages of the stochastic heat equation via Malliavin-Stein's method. In preparation, 2020.

[7] L. Chen, Le, Y. Hu, Yaozhong and D. Nualart: Two-point correlation function and Feynman-Kac formula for the stochastic heat equation. Potential Anal. 46 (2017), 779-797.

[8] L. Chen, Y. Hu and D. Nualart: Regularity and strict positivity of densities for the nonlinear stochastic heat equation. To appear in Memoirs of the AMS, 2019.

[9] L. Chen and J. Huang: Regularity and strict positivity of densities for the stochastic heat equation on $\mathbb{R}^{d}$. arXiv:1902.02382, 2019.

[10] R. C. Dalang: Extending the martingale measure stochastic. Electronic Journal of Probability 4 (1999), 29 pp.

[11] Y. Hu, J. Huang, D. Nualart and S. Tindel: Stochastic heat equations with general multiplicative Gaussian noises: Hölder continuity and intermittency, Electronic Journal of Probability 20 (2015), paper no. 55.

[12] Y. Hu and D. Nualart, David: Stochastic heat equation driven by fractional noise and local time. Probab. Theory Related Fields, 143 (2009), 285-328.

[13] J. Huang, K. Lê and D. Nualart: Large time asymptotics for the parabolic Anderson model driven by spatially correlated noise, Annales de l'Institut Henri Poincaré, Probabilités et Statistiques 53 (2017), 1305-1340.

[14] J. Huang, K. Lê and D. Nualart: Large time asymptotics for the parabolic Anderson model driven byspace and time correlated noise. Stoch. Partial Differ. Equ. Anal. Comput. 5 (2017), no. 4, 614-651.

[15] J. Huang, D. Nualart and L. Viitasaari: A central limit theorem for the stochastic heat equation. Preprint, 2018.

[16] J. Huang, D. Nualart, L. Viitasaari and G. Zheng: Gaussian fluctuations for the stochastic heat equation with colored noise. Preprint, 2019.

[17] D. Nualart: The Malliavin calculus and related topics, 2006, Springer. 
[18] D. Nualart and E. Nualart: Introduction to Malliavin calculus, 2018, Cambridge University Press.

[19] M. Sanz-Solé and M. Sarrà: Hölder continuity for the stochastic heat equation with spatially correlated noise. In: Seminar on Stochastic Analysis, Random Fields and Applications, III (Ascona, 1999), 259-268, Progr. Probab., 52 (2002), Birkhäuser, Basel.

[20] J. B. Walsh: An introduction to stochastic partial differential equations. In: École d'Été de Probabilités de Saint Flour XIV-1984, 265-439, 1986, Springer.

University of Kansas, Department of Mathematics, USA

E-mail address: sefika.kuzgun@ku.edu

University of Kansas, Department of Mathematics, USA

E-mail address: nualart@ku.edu 